\title{
New stabilized discretizations for poroelasticity and the Stokes' equations
}

\author{
C. Rodrigo ${ }^{\mathrm{a}, *}$, X. Hu ${ }^{\mathrm{b}}$, P. Ohm ${ }^{\mathrm{b}}$, J.H. Adler ${ }^{\mathrm{b}}$, F.J. Gaspar ${ }^{\mathrm{c}}$, L.T. Zikatanov ${ }^{\mathrm{d}, \mathrm{e}}$ \\ ${ }^{a}$ Departamento de Matemática Aplicada, IUMA, Universidad de Zaragoza, Zaragoza, Spain \\ ${ }^{\mathrm{b}}$ Department of Mathematics, Tufts University, Medford, Massachusetts 02155, USA \\ ${ }^{\mathrm{c}}$ CWI, Centrum Wiskunde \& Informatica, Science Park 123, 1090 Amsterdam, The Netherlands \\ ${ }^{\mathrm{d}}$ Department of Mathematics, The Pennsylvania State University, University Park, PA 16802, USA \\ e Institute for Mathematics and Informatics, Bulgarian Academy of Sciences, Sofia, Bulgaria
}

Received 15 June 2017; received in revised form 14 May 2018; accepted 2 July 2018

Available online 11 July 2018

\begin{abstract}
In this work, we consider the popular P1-RT0-P0 discretization of the three-field formulation of Biot's consolidation problem. Since this finite-element formulation is not uniformly stable with respect to the physical parameters, several issues arise in numerical simulations. For example, when the permeability is small with respect to the mesh size, volumetric locking may occur. To alleviate such problems, we consider a well-known stabilization technique with face bubble functions. We then design a perturbation of the bilinear form, which allows for local elimination of the bubble functions. We further prove that such perturbation is consistent and the resulting scheme has optimal approximation properties for both Biot's model as well as the Stokes' equations. For the former, the number of degrees of freedom is the same as for the classical P1-RT0-P0 discretization and for the latter (Stokes' equations) the number of degrees of freedom is the same as for a P1-P0 discretization. We present numerical tests confirming the theoretical results for the poroelastic and the Stokes' test problems.
\end{abstract}

(C) 2018 Elsevier B.V. All rights reserved.

Keywords: Stable finite elements; Poroelasticity; Stokes' equations

\section{Introduction}

The interaction between the deformation and fluid flow in a fluid-saturated porous medium is the object of study in poroelasticity theory. Such coupling has been modeled in the early one-dimensional work of Terzaghi [1]. A more general three-dimensional mathematical formulation was then established by Maurice Biot in several pioneering publications (see [2] and [3]). Biot's models are widely used nowadays in the modeling of many applications in different fields, ranging from geomechanics and petroleum engineering, to biomechanics. The existence and

\footnotetext{
* Corresponding author.

E-mail addresses: carmenr@unizar.es (C. Rodrigo), xiaozhe.hu@tufts.edu (X. Hu), peter.ohm@tufts.edu (P. Ohm), james.adler@tufts.edu (J.H. Adler), F.J.Gaspar@cwi.nl (F.J. Gaspar), ludmil@psu.edu (L.T. Zikatanov).
} 
uniqueness of the solution for these problems have been investigated by Showalter in [4] and by Zenisek in [5]. Regarding the numerical simulation of the poroelasticity equations, there have been numerous contributions using finite-difference schemes [6,7] and finite-volume methods (see [8,9] for recent developments). Finite-element methods, which are the subject of this work, have also been considered (see for example the monograph by Lewis and Schrefler [10] and the references therein).

Stable finite-element schemes are constructed by either choosing discrete spaces satisfying appropriate inf-sup (or LBB) conditions, or applying suitable stabilization techniques to unstable finite-element pairs. For two-field (displacement-pressure) formulations of Biot's problem, the classical Taylor-Hood elements belong to the first class [11-13], as well as the MINI element [14]. On the other hand, a stabilized discretization based on linear finite elements for both displacements and pressure was recently analyzed in [14], and belongs to the second type. Regarding three-field formulations, which include the Darcy velocity as an unknown, several conforming and nonconforming discretizations involving Stokes-stable finite-element spaces were proposed in recent years. For instance, a stable finite-element method based on non-conforming Crouzeix-Raviart finite elements for the displacements, lowest order Raviart-Thomas-Nédélec elements for the Darcy velocity, and piecewise constants for the pressure was proposed in [15]. In [16], a family of parameter-robust three-field finite-element schemes were proposed and analyzed and a general theory for the error analysis was introduced. Additionally, a novel three-field formulation based on displacement, pressure, and total pressure was proposed in [17] with error estimates independent of the Lamé constants, yielding a locking-free approach. Furthermore, in [18], one finds a parameter-robust error analysis and optimal preconditioning techniques for several discretizations of three-field formulations for Biot's model. For a four-field formulation of the problem, which includes the stress tensor, the fluid flux, the solid displacement, and the pore pressure as unknowns, a stable approach is proposed in [19]. In that work, two sets of mixed finite elements, one for linear elasticity and one for mixed Poisson, are coupled for the spatial discretization. Finally, stable iterative schemes, such as the fixed stress method, have also been developed and analyzed for formulations of Biot's model (e.g., [20,21]).

This paper focuses on the three-field formulation, which has received a lot of attention from the point of view of novel discretizations [22-24], as well as for the design of efficient solvers [25-27]. Because of its application to existing reservoir engineering simulators, one of the most frequently considered schemes is a three-field formulation based on piecewise linear elements for displacements, Raviart-Thomas-Nédélec elements for the fluid flux, and piecewise constants for the pressure. This element, however, does not satisfy an inf-sup condition uniformly with respect to the physical parameters of the problem. Thus, we propose a stabilization of this popular element which gives rise to uniform error bounds, keeping the same number of degrees of freedom as in the original method.

A consequence of our analysis is that a new stable scheme for the Stokes' equations is derived. The resulting method can be seen as a perturbation of the well-known unstable pair based on piecewise linear and piecewise constant elements for velocities and pressure, respectively (P1-P0). However, this perturbation yields a stable finite-element pair for Stokes, which has the lowest possible number of degrees of freedom.

The rest of the paper is organized as follows. Section 2 is devoted to describing Biot's problem and, in particular, the considered three-field formulation and its discretization. A numerical example is given, illustrating the difficulties that appear when using the standard, unstabilized, approach. In Section 3, we introduce the stabilized scheme in which we consider the enrichment of the piecewise linear continuous finite-element space with edge/face (2D/3D) bubble functions. Section 4 is devoted to the local elimination of the bubbles to maintain the same number of degrees of freedom as in the original scheme. The well-posedness of the resulting scheme, as well as the corresponding error analysis are also provided here. In Section 5, we present the Stokes-stable finite-element method based on P1-P0 finite elements obtained by following the same strategy as presented in the previous sections for poroelasticity. Finally, in Section 6, we confirm the uniform convergence properties of the stabilized schemes for both poroelasticity and Stokes' equations through some numerical tests.

\section{Preliminaries: model problem and notation}

We consider the quasi-static Biot's model for consolidation in a linearly elastic, homogeneous, and isotropic porous medium saturated by an incompressible Newtonian fluid. According to Biot's theory [2], the mathematical model of the consolidation process is described by the following system of partial differential equations (PDEs) in a domain 
$\Omega \subset \mathbb{R}^{d}, d=2,3$ with sufficiently smooth boundary $\Gamma=\partial \Omega$ :

$$
\begin{aligned}
\text { equilibrium equation: } & -\operatorname{div} \boldsymbol{\sigma}^{\prime}+\alpha \nabla p=\rho \boldsymbol{g}, \quad \text { in } \Omega, \\
\text { constitutive equation: } & \boldsymbol{\sigma}^{\prime}=2 \mu \boldsymbol{\varepsilon}(\boldsymbol{u})+\lambda \operatorname{div}(\boldsymbol{u}) \boldsymbol{I}, \quad \text { in } \Omega, \\
\text { compatibility condition: } & \boldsymbol{\varepsilon}(\boldsymbol{u})=\frac{1}{2}\left(\nabla \boldsymbol{u}+\nabla \boldsymbol{u}^{t}\right), \quad \text { in } \Omega, \\
\text { Darcy's law: } & \boldsymbol{w}=-\frac{1}{\mu_{f}} \boldsymbol{K}\left(\nabla p-\rho_{f} \boldsymbol{g}\right), \quad \text { in } \Omega,
\end{aligned}
$$

continuity equation: $\quad \frac{\partial}{\partial t}\left(\frac{1}{M} p+\alpha \operatorname{div} \boldsymbol{u}\right)+\operatorname{div} \boldsymbol{w}=f, \quad$ in $\Omega$,

where $\lambda$ and $\mu$ are the Lamé coefficients, $M$ is the Biot modulus, and $\alpha=1-\frac{K_{b}}{K_{s}}$ is the Biot-Willis constant. Here, $K_{b}$ and $K_{s}$ denote the drained and the solid phase bulk moduli. As is customary, $\boldsymbol{K}$ stands for the absolute permeability tensor, $\mu_{f}$ is the viscosity of the fluid, and $\boldsymbol{I}$ is the identity tensor. The unknown functions are the displacement vector $\boldsymbol{u}$ and the pore pressure $p$. The effective stress tensor and the strain tensor are denoted by $\boldsymbol{\sigma}^{\prime}$ and $\boldsymbol{\varepsilon}$, respectively. The percolation velocity of the fluid, or Darcy's velocity, relative to the soil is denoted by $\boldsymbol{w}$ and the vector-valued function $\boldsymbol{g}$ represents the gravitational force. The bulk density is $\rho=\phi \rho_{f}+(1-\phi) \rho_{s}$, where $\rho_{s}$ and $\rho_{f}$ are the densities of solid and fluid phases and $\phi$ is the porosity. Finally, the source term $f$ represents a forced fluid extraction or injection process.

Our focus here is on the so-called three-field formulation in which Darcy's velocity, $\boldsymbol{w}$, is also a primary unknown in addition to $\boldsymbol{u}$ and $p$. As a result, we have the following system of PDEs:

$$
\begin{aligned}
& -\operatorname{div} \boldsymbol{\sigma}^{\prime}+\alpha \nabla p=\rho \boldsymbol{g}, \quad \text { where } \quad \boldsymbol{\sigma}^{\prime}=2 \mu \boldsymbol{\varepsilon}(\boldsymbol{u})+\lambda \operatorname{div}(\boldsymbol{u}) \boldsymbol{I}, \\
& \mathbf{K}^{-1} \mu_{f} \boldsymbol{w}+\nabla p=\rho_{f} \boldsymbol{g}, \\
& \frac{\partial}{\partial t}\left(\frac{1}{M} p+\alpha \operatorname{div} \boldsymbol{u}\right)+\operatorname{div} \boldsymbol{w}=f .
\end{aligned}
$$

This system is often subject to the following set of boundary conditions:

$$
\begin{aligned}
& p=0, \quad \text { for } \quad x \in \bar{\Gamma}_{t}, \quad \boldsymbol{\sigma}^{\prime} \boldsymbol{n}=\mathbf{0}, \quad \text { for } \quad x \in \Gamma_{t}, \\
& \boldsymbol{u}=\mathbf{0}, \quad \text { for } \quad x \in \bar{\Gamma}_{c}, \quad \frac{\partial p}{\partial \boldsymbol{n}}=0, \quad \text { for } \quad x \in \Gamma_{c},
\end{aligned}
$$

where $\boldsymbol{n}$ is the outward unit normal to the boundary, $\bar{\Gamma}=\bar{\Gamma}_{t} \cup \bar{\Gamma}_{c}$, with $\Gamma_{t}$ and $\Gamma_{c}$ being open (with respect to $\Gamma$ ) subsets of $\Gamma$ with nonzero measure. In the following, we omit the symbol ".-" over $\Gamma_{t}$ and $\Gamma_{c}$ as it will be clear from the context that the essential boundary conditions are imposed on closed subsets of $\Gamma$. Non-homogeneous boundary conditions are also of interest. Note that Dirichlet boundary conditions for $p$ imply Neumann boundary conditions for $\boldsymbol{w}$ and vice-versa.

The initial condition at $t=0$ is given by,

$$
\left(\frac{1}{M} p+\alpha \operatorname{div} u\right)(x, 0)=0, x \in \Omega
$$

which yields the following mixed formulation of Biot's three-field consolidation model:

For each $t \in(0, T]$, find $(\boldsymbol{u}(t), \boldsymbol{w}(t), p(t)) \in \boldsymbol{V} \times \boldsymbol{W} \times Q$ such that

$$
\begin{aligned}
& a(\boldsymbol{u}, \boldsymbol{v})-(\alpha p, \operatorname{div} \boldsymbol{v})=(\rho \boldsymbol{g}, \boldsymbol{v}), \quad \forall \boldsymbol{v} \in \boldsymbol{V}, \\
& \left(\boldsymbol{K}^{-1} \mu_{f} \boldsymbol{w}, \boldsymbol{r}\right)-(p, \operatorname{div} \boldsymbol{r})=\left(\rho_{f} \boldsymbol{g}, \boldsymbol{r}\right), \quad \forall \boldsymbol{r} \in \boldsymbol{W}, \\
& \left(\frac{1}{M} \frac{\partial p}{\partial t}, q\right)+\left(\alpha \operatorname{div} \frac{\partial \boldsymbol{u}}{\partial t}, q\right)+(\operatorname{div} \boldsymbol{w}, q)=(f, q), \quad \forall q \in Q,
\end{aligned}
$$

where,

$$
a(\boldsymbol{u}, \boldsymbol{v})=2 \mu \int_{\Omega} \boldsymbol{\varepsilon}(\boldsymbol{u}): \boldsymbol{\varepsilon}(\boldsymbol{v})+\lambda \int_{\Omega} \operatorname{div} \boldsymbol{u} \operatorname{div} \boldsymbol{v},
$$


corresponds to linear elasticity. The function spaces used in the variational form are

$$
\begin{aligned}
& \boldsymbol{V}=\left\{\boldsymbol{u} \in \boldsymbol{H}^{1}(\Omega)|\boldsymbol{u}|_{\Gamma_{c}}=\mathbf{0}\right\}, \\
& \boldsymbol{W}=\left\{\boldsymbol{w} \in \boldsymbol{H}(\operatorname{div}, \Omega)|(\boldsymbol{w} \cdot \boldsymbol{n})|_{\Gamma_{c}}=0\right\}, \\
& Q=L^{2}(\Omega),
\end{aligned}
$$

where $\boldsymbol{H}^{1}(\Omega)$ is the space of square integrable vector-valued functions whose first derivatives are also square integrable, and $\boldsymbol{H}(\operatorname{div}, \Omega)$ contains the square integrable vector-valued functions with square integrable divergence.

We recall that the well-posedness of the continuous problem was established by Showalter [4], and, for the threefield formulation by Lipnikov [28]. Next, we focus on the behavior of some classical discretizations of Biot's model.

\subsection{Discretizations}

First, we partition the domain $\Omega$ into $n$-dimensional simplices and denote the resulting partition with $\mathcal{T}_{h}$, i.e., $\bar{\Omega}=$ $\cup_{T \in \mathcal{T}_{h}} \bar{T}$. Further, with every simplex $T \in \mathcal{T}_{h}$, we associate two quantities which characterize its shape: the diameter of $T, h_{T}=\operatorname{diam}(T)$, and the radius, $\rho_{T}$, of the $n$-dimensional ball inscribed in $T$. The simplicial mesh is shape regular if and only if $h_{T} / \rho_{T} \lesssim 1$ uniformly with respect to $T$.

With the partitioning, $\mathcal{T}_{h}$, we associate a triple of piecewise polynomial, finite-dimensional spaces,

$$
\boldsymbol{V}_{h} \subset \boldsymbol{V}, \quad \boldsymbol{W}_{h} \subset \boldsymbol{W}, \quad Q_{h} \subset Q .
$$

While we specify two choices of the space $\boldsymbol{V}_{h}$ later, we fix $\boldsymbol{W}_{h}$ and $Q_{h}$ as follows,

$$
\begin{aligned}
& \boldsymbol{W}_{h}=\left\{\boldsymbol{w}_{h} \in \boldsymbol{W}\left|\boldsymbol{w}_{h}\right|_{T}=\boldsymbol{a}+\eta \mathbf{x}, \boldsymbol{a} \in \mathbb{R}^{d}, \eta \in \mathbb{R}, \quad \forall T \in \mathcal{T}_{h}\right\}, \\
& Q_{h}=\left\{q_{h} \in Q\left|q_{h}\right|_{T} \in \mathbb{P}_{0}(T), \quad \forall T \in \mathcal{T}_{h}\right\},
\end{aligned}
$$

where $\mathbb{P}_{0}(T)$ is the one-dimensional space of constant functions on $T$. We note that the inclusions listed in (16) imply that the elements of $\boldsymbol{V}_{h}$ are continuous on $\Omega$, the functions in $\boldsymbol{W}_{h}$ have continuous normal components across element boundaries, and that the functions in $Q_{h}$ are in $L^{2}(\Omega)$. This choice of $\boldsymbol{W}_{h}$ is the standard lowest order RaviartThomas-Nédélec space (RT0) and $Q_{h}$ is the piecewise constant space (P0).

Finally, using backward Euler as a time discretization on a time interval $\left(0, t_{\max }\right]$ with constant time-step size $\tau$, the discrete scheme corresponding to the three-field formulation (12)-(14) reads:

Find $\left(\boldsymbol{u}_{h}^{m}, \boldsymbol{w}_{h}^{m}, p_{h}^{m}\right) \in \boldsymbol{V}_{h} \times \boldsymbol{W}_{h} \times Q_{h}$ such that

$$
\begin{aligned}
& a\left(\boldsymbol{u}_{h}^{m}, \boldsymbol{v}_{h}\right)-\left(\alpha p_{h}^{m}, \operatorname{div} \boldsymbol{v}_{h}\right)=\left(\rho \boldsymbol{g}, \boldsymbol{v}_{h}\right), \quad \forall \boldsymbol{v}_{h} \in \boldsymbol{V}_{h}, \\
& \tau\left(\boldsymbol{K}^{-1} \mu_{f} \boldsymbol{w}_{h}^{m}, \boldsymbol{r}_{h}\right)-\tau\left(p_{h}^{m}, \operatorname{div} \boldsymbol{r}_{h}\right)=\tau\left(\rho_{f} \boldsymbol{g}, \boldsymbol{r}_{h}\right), \quad \forall \boldsymbol{r}_{h} \in \boldsymbol{W}_{h}, \\
& \left(\frac{1}{M} p_{h}^{m}, q_{h}\right)+\left(\alpha \operatorname{div} \boldsymbol{u}_{h}^{m}, q_{h}\right)+\tau\left(\operatorname{div} \boldsymbol{w}_{h}^{m}, q_{h}\right)=\left(\tilde{f}, q_{h}\right), \quad \forall q_{h} \in Q_{h},
\end{aligned}
$$

where $\left(\tilde{f}, q_{h}\right)=\tau\left(f, q_{h}\right)+\left(\frac{1}{M} p_{h}^{m-1}, q_{h}\right)+\left(\alpha \operatorname{div} \boldsymbol{u}_{h}^{m-1}, q_{h}\right)$, and,

$$
\left(\boldsymbol{u}_{h}^{m}, \boldsymbol{w}_{h}^{m}, p_{h}^{m}\right) \approx\left(\boldsymbol{u}\left(\cdot, t_{m}\right), \boldsymbol{w}\left(\cdot, t_{m}\right), p\left(\cdot, t_{m}\right)\right), \quad t_{m}=m \tau, m=1,2, \ldots
$$

Note that (18) has been scaled by $\tau$ for symmetry reasons.

\subsection{Effects of permeability on the error of approximation}

For $\boldsymbol{V}_{h}$, we start with a popular finite-element approximation for (12)-(14) by choosing

$$
\boldsymbol{V}_{h}=\boldsymbol{V}_{h, 1}, \quad \text { with } \quad \boldsymbol{V}_{h, 1}:=\left\{\boldsymbol{v}_{h} \in \boldsymbol{V}\left|\boldsymbol{v}_{h}\right|_{T} \in\left[\mathbb{P}_{1}(T)\right]^{d}, \text { for all } T \in \mathcal{T}_{h}\right\},
$$

where $\mathbb{P}_{1}(T)$ is the space of linear polynomials on $T \in \mathcal{T}_{h}$. Then, $V_{h, 1}$ is the space of piecewise linear (with respect to $\mathcal{T}_{h}$ ), continuous vector-valued functions. For uniformly positive definite permeability tensor, $\boldsymbol{K}$, such choice of spaces has been successfully employed for numerical simulations of Biot's consolidation model (see $[23,28]$ ). However, the heuristic considerations that expose some of the issues with this discretization are observed in cases when $\boldsymbol{K} \rightarrow \mathbf{0}$. In such cases, $\boldsymbol{w} \rightarrow \boldsymbol{O}$ and the discrete problem approaches a P1-P0 discretization of the Stokes' equation. As it is well 
Table 2.1

Energy norm and $L^{2}$-norm for displacement and pressure errors, respectively, for various values of hydraulic conductivity, $\kappa=\left(k / \mu_{f}\right)$, and number of elements in each direction, $N$. Results confirm poor approximation when $\kappa / h$ is small.

\begin{tabular}{lllllll}
\hline & & $N=8$ & $N=16$ & $N=32$ & $N=64$ & $N=128$ \\
\hline \multirow{2}{*}{$=10^{-4}$} & $\left\|\boldsymbol{u}-\boldsymbol{u}_{h}\right\|_{A}$ & 0.0209 & 0.0089 & 0.0043 & 0.0022 & 0.0011 \\
& $\left\|p-p_{h}\right\|_{L^{2}}$ & 0.0535 & 0.0088 & 0.0015 & 0.0003 & $7.38 \times 10^{-5}$ \\
\hline \multirow{2}{*}{$\kappa=10^{-6}$} & $\left\|\boldsymbol{u}-\boldsymbol{u}_{h}\right\|_{A}$ & 0.0477 & 0.0271 & 0.0060 & 0.0022 & 0.0011 \\
& $\left\|p-p_{h}\right\|_{L^{2}}$ & 0.3277 & 0.3199 & 0.0763 & 0.0099 & 0.0012 \\
\hline \multirow{2}{*}{$\kappa=10^{-8}$} & $\left\|\boldsymbol{u}-\boldsymbol{u}_{h}\right\|_{A}$ & 0.0503 & 0.0497 & 0.0418 & 0.0147 & 0.0019 \\
& $\left\|p-p_{h}\right\|_{L^{2}}$ & 0.3553 & 0.7157 & 1.1509 & 0.6537 & 0.1152 \\
\hline \multirow{2}{*}{$\kappa=10^{-10}$} & $\left\|\boldsymbol{u}-\boldsymbol{u}_{h}\right\|_{A}$ & 0.0503 & 0.0503 & 0.0501 & 0.0484 & 0.0330 \\
& $\left\|p-p_{h}\right\|_{L^{2}}$ & 0.3550 & 0.7271 & 1.4576 & 2.7836 & 3.4508 \\
\hline
\end{tabular}

known, the element pair, $\boldsymbol{V}_{h, 1} \times Q_{h}$, does not satisfy the inf-sup condition and is unstable for the Stokes' problem. In fact, on a uniform grid in $2 D$, it is easy to prove that volumetric locking occurs, namely, that the only divergence-free function from $V_{h, 1}$ is the zero function. More precisely,

$$
\operatorname{dim}\left(Q_{h}\right)>\operatorname{dim} \boldsymbol{V}_{h}>\operatorname{dim} \operatorname{Range}\left(\operatorname{div}_{h}\right), \quad \operatorname{div}_{h}=\left.\operatorname{div}\right|_{V_{h}} .
$$

These inequalities imply that $\operatorname{div}_{h}$ is not an onto operator, and, hence, the pair of spaces violates the inf-sup condition associated with the discrete Stokes' problem. More details on this undesirable phenomenon for Stokes are found in the classical monograph [29] and also in [30, pp. 45-100] and [31].

Here, we demonstrate numerically that for Biot's model, the error in the finite-element approximation does not decrease when the permeability is small relative to the mesh size. We consider $\Omega=(0,1) \times(0,1)$, and approximate (12)-(14) subject to homogeneous Dirichlet boundary conditions for $\boldsymbol{u}$, and Neumann boundary conditions for $p$ for the rest of $\Gamma$. We cover $\Omega$ with a uniform triangular grid by dividing an $N \times N$ uniform square mesh into right triangles, where the mesh spacing is defined by $h=\frac{1}{N}$. The material parameters are $\lambda=2, \mu=1, \mu_{f}=1, \alpha=1$, and $M=10^{6}$. We consider a diagonal permeability tensor $\boldsymbol{K}=k \boldsymbol{I}$ with constant $k$, and introduce the hydraulic conductivity, $\kappa=k / \mu_{f}$. The other data is set so that the exact solution is given by

$$
\begin{aligned}
& \boldsymbol{u}(x, y, t)=\operatorname{curl} \varphi=\left(\begin{array}{c}
\partial_{y} \varphi \\
-\partial_{x} \varphi
\end{array}\right), \quad \varphi(x, y)=[x y(1-x)(1-y)]^{2}, \\
& p(x, y, t)=1 .
\end{aligned}
$$

Finally, we set $\tau=1$ and $t_{\max }=1$, so that we only perform one time step.

As seen in Table 2.1 the energy norm $\left(\|v\|_{A}^{2}:=a(\boldsymbol{v}, \boldsymbol{v})\right.$ for $\left.\boldsymbol{v} \in \boldsymbol{V}\right)$ for the displacement errors and the $L^{2}$-norm for pressure errors do not decrease until the mesh size is sufficiently small (compared with the permeability). Thus for small permeabilities, this could result in expensive discretizations which are less applicable to practical situations.

\section{Stabilization and perturbation of the bilinear form}

To resolve the above issue, we introduce a well-known stabilization technique based on enrichment of the piecewise linear continuous finite-element space, $\boldsymbol{V}_{h, 1}$, with edge/face (2D/3D) bubble functions (see [32, pp. 145-149]). The discretization described below is based on a Stokes-stable pair of spaces $\left(\boldsymbol{V}_{h}, Q_{h}\right)$ with $\boldsymbol{V}_{h} \supset \boldsymbol{V}_{h, 1}$. As we show later, in Section 4, this stabilization gives a proper finite-element approximation of the solution of Biot's model independently of the size of the permeability, $\boldsymbol{K}$, or hydraulic conductivity, $\kappa$.

\subsection{Stabilization by face bubbles}

To define the enriched space, following [32], consider the set of $(d-1)$ dimensional faces from $\mathcal{T}_{h}$ and denote this set by $\mathcal{E}=\mathcal{E}^{o} \cup \mathcal{E}^{\partial}$, where $\mathcal{E}^{o}$ is the set of interior faces (shared by two elements) and $\mathcal{E}^{\partial}$ is the set of faces on the boundary. In addition, $\mathcal{E}^{\Gamma_{t}}$ is the set of faces on the boundary $\Gamma_{t}$ and $\mathcal{E}^{o, t}=\mathcal{E}^{o} \cap \mathcal{E}^{\Gamma_{t}}$. Note, if $\Gamma_{t}=\partial \Omega$ (pure traction boundary condition), then $\mathcal{E}^{\Gamma_{t}}=\mathcal{E}^{\partial}$ and $\mathcal{E}^{o, t}=\mathcal{E}$. For any face $e \in \mathcal{E}^{o}$, such that $e \in \partial T$, and $T \in \mathcal{T}_{h}$, let $\boldsymbol{n}_{e, T}$ be the outward (with respect to $T$ ) unit normal vector to $e$. With every face $e \in \mathcal{E}^{o}$, we also associate a unit vector $\boldsymbol{n}_{e}$ which 
is orthogonal to it. Clearly, if $e \in \partial T$ we have $\boldsymbol{n}_{e}= \pm \boldsymbol{n}_{e, T}$. For the boundary faces $e \in \mathcal{E}^{\partial}$, we always set $\boldsymbol{n}_{e}=\boldsymbol{n}_{e, T}$, where $T$ is the unique element for which we have $e \subset \partial T$. For the interior faces, the particular direction of $\boldsymbol{n}_{e}$ is not important, although it is important that this direction is fixed. More precisely,

$$
\boldsymbol{n}_{e}=\boldsymbol{n}_{e, T^{+}}=-\boldsymbol{n}_{e, T^{-}} \quad \text { if } \quad e=T^{+} \cap T^{-}, \quad \text { and } \quad T^{ \pm} \in \mathcal{T}_{h} .
$$

Further, with every face $e \in \mathcal{E}, e=T^{+} \cap T^{-}$, we associate a vector-valued function $\Phi_{e}$,

$$
\boldsymbol{\Phi}_{e}=\varphi_{e} \boldsymbol{n}_{e}, \quad \text { with }\left.\quad \varphi_{e}\right|_{T^{ \pm}}=\varphi_{e, T^{ \pm}}, \quad \text { and } \quad \varphi_{e, T^{ \pm}}=\prod_{k=1, k \neq j^{ \pm}}^{d+1} \lambda_{k, T^{ \pm}},
$$

where $\lambda_{k, T^{ \pm}}, k=1, \ldots,(d+1)$ are barycentric coordinates on $T^{ \pm}$and $j^{ \pm}$is the vertex opposite to the face $e$ in $T^{ \pm}$. We note that $\boldsymbol{\Phi}_{e} \in \boldsymbol{V}$ is a continuous piecewise polynomial function of degree $d$.

Finally, the stabilized finite-element space $V_{h}$ is defined as

$$
\boldsymbol{V}_{h}=\boldsymbol{V}_{h, 1} \oplus \boldsymbol{V}_{b}, \quad \boldsymbol{V}_{b}=\operatorname{span}\left\{\boldsymbol{\Phi}_{e}\right\}_{e \in \mathcal{E}^{o, t}} .
$$

The degrees of freedom associated with $\boldsymbol{V}_{h}$ are the values at the vertices of $\mathcal{T}_{h}$ and the total flux through $e \in \mathcal{E}^{o, t}$ of $\left(I-\Pi_{1}\right) \boldsymbol{v}_{h}$, where $\Pi_{1}$ is the standard piecewise linear interpolant, $\Pi_{1}: C(\bar{\Omega}) \mapsto \boldsymbol{V}_{h, 1}$. Then, the canonical interpolant, $\Pi: C(\bar{\Omega}) \mapsto V_{h}$, is defined as:

$$
\Pi v=\Pi_{1} v+\sum_{e \in \mathcal{E}^{o, t}} v_{e} \Phi_{e}, \quad v_{e}=\frac{1}{|e|} \int_{e}\left(I-\Pi_{1}\right) v .
$$

With this choice of $\boldsymbol{V}_{h}$, the variational form, (17)-(19), remains the same and we have the following block form of the discrete problem:

$$
\mathcal{A}\left(\begin{array}{l}
\boldsymbol{U}_{b} \\
\boldsymbol{U}_{l} \\
\boldsymbol{W} \\
\boldsymbol{P}
\end{array}\right)=\boldsymbol{b}, \quad \text { with } \mathcal{A}=\left(\begin{array}{cccc}
A_{b b} & A_{b l} & 0 & G_{b} \\
A_{b l}^{T} & A_{l l} & 0 & G_{l} \\
0 & 0 & \tau M_{w} & \tau G \\
G_{b}^{T} & G_{l}^{T} & \tau G^{T} & -M_{p}
\end{array}\right),
$$

where $\boldsymbol{U}_{b}, \boldsymbol{U}_{l}, \boldsymbol{W}$ and $\boldsymbol{P}$ are the unknown vectors for the bubble components of the displacement, the piecewise linear components of the displacement, the Darcy velocity, and the pressure, respectively. The blocks in the definition of $\mathcal{A}$ correspond to the following bilinear forms:

$$
\begin{aligned}
& a\left(\boldsymbol{u}_{h}^{b}, \boldsymbol{v}_{h}^{b}\right) \rightarrow A_{b b}, \quad a\left(\boldsymbol{u}_{h}^{l}, \boldsymbol{v}_{h}^{b}\right) \rightarrow A_{b l}, \quad a\left(\boldsymbol{u}_{h}^{l}, \boldsymbol{v}_{h}^{l}\right) \rightarrow A_{l l}, \\
& -\left(\alpha p_{h}, \operatorname{div} \boldsymbol{v}_{h}^{b}\right) \rightarrow G_{b}, \quad-\left(\alpha p_{h}, \operatorname{div} \boldsymbol{v}_{h}^{l}\right) \rightarrow G_{l}, \quad-\left(p_{h}, \operatorname{div} \boldsymbol{r}_{h}\right) \rightarrow G, \\
& \left(K^{-1} \mu_{f} \boldsymbol{w}_{h}, \boldsymbol{r}_{h}\right) \rightarrow M_{w}, \quad\left(\frac{1}{M} p_{h}, q_{h}\right) \rightarrow M_{p},
\end{aligned}
$$

where $\boldsymbol{u}_{h}=\boldsymbol{u}_{h}^{l}+\boldsymbol{u}_{h}^{b}, \boldsymbol{u}_{h}^{l} \in \boldsymbol{V}_{h, 1}, \boldsymbol{u}_{h}^{b} \in \boldsymbol{V}_{b}$, and an analogous decomposition for $\boldsymbol{v}_{h}$.

Next, we define the following notion of stability for discretizations of Biot's model needed for the analysis.

Definition 3.1. The triple of spaces $\left(\widetilde{\boldsymbol{V}}_{h}, \widetilde{\boldsymbol{W}}_{h}, \widetilde{Q}_{h}\right)$ is Stokes-Biot stable if and only if the following conditions are satisfied:

- $a\left(\boldsymbol{u}_{h}, \boldsymbol{v}_{h}\right) \leq C_{\boldsymbol{V}}\left\|\boldsymbol{u}_{h}\right\|_{1}\left\|\boldsymbol{v}_{h}\right\|_{1}$, for all $\underline{\boldsymbol{u}}_{h} \in \widetilde{\boldsymbol{V}}_{h}, \boldsymbol{v}_{h} \in \widetilde{\boldsymbol{V}}_{h}$;

- $a\left(\boldsymbol{u}_{h}, \boldsymbol{u}_{h}\right) \geq \alpha_{V}\left\|\boldsymbol{u}_{h}\right\|_{1}^{2}$, for all $\boldsymbol{u}_{h} \in \widetilde{\boldsymbol{V}}_{h}$;

- The pair of spaces $\left(\widetilde{\boldsymbol{W}}_{h}, \widetilde{Q}_{h}\right)$ is Poisson stable, i.e., it satisfies stability and continuity conditions required by the mixed discretization of the Poisson equation;

- The pair of spaces $\left(\widetilde{\boldsymbol{V}}_{h}, \widetilde{Q}_{h}\right)$ is Stokes stable.

Here, $\|\cdot\|_{1}$ and $\|\cdot\|$ denote the standard $\boldsymbol{H}^{1}$ norm and $L^{2}$ norm, respectively.

As mentioned earlier, the authors in [16] also propose a parameter-robust stable scheme for Biot's system based on the conditions above. Following the same idea, here, we introduce a norm on $\boldsymbol{V}_{h} \times \boldsymbol{W}_{h} \times Q_{h}$ :

$$
\left\|\left(\boldsymbol{u}_{h}, \boldsymbol{w}_{h}, p_{h}\right)\right\|:=\left[\left\|\boldsymbol{u}_{h}\right\|_{A}+\tau\left\|\boldsymbol{w}_{h}\right\|_{K^{-1} \mu_{f}}^{2}+\tau^{2} \xi^{-1}\left\|\operatorname{div} \boldsymbol{w}_{h}\right\|^{2}+\xi\left\|p_{h}\right\|^{2}\right]^{1 / 2},
$$

where $\zeta=\sqrt{\lambda+2 \mu / d}, \xi=\frac{\alpha^{2}}{\zeta^{2}}+\frac{1}{M}$, and $\|\boldsymbol{r}\|_{K^{-1} \mu_{f}}:=\left(K^{-1} \mu_{f} \boldsymbol{r}, \boldsymbol{r}\right)^{1 / 2}$. 
Further, we associate a composite bilinear form on the space, $\boldsymbol{V}_{h} \times \boldsymbol{W}_{h} \times Q_{h}$,

$$
\begin{gathered}
B\left(\boldsymbol{u}_{h}, \boldsymbol{w}_{h}, p_{h} ; \boldsymbol{v}_{h}, \boldsymbol{r}_{h}, q_{h}\right):=a\left(\boldsymbol{u}_{h}, \boldsymbol{v}_{h}\right)-\left(\alpha p_{h}, \operatorname{div} \boldsymbol{v}_{h}\right)+\tau\left(K^{-1} \mu_{f} \boldsymbol{w}_{h}, \boldsymbol{r}_{h}\right)-\tau\left(p_{h}, \operatorname{div} \boldsymbol{r}_{h}\right) \\
-\left(\frac{1}{M} p_{h}, q_{h}\right)-\left(\alpha \operatorname{div} \boldsymbol{u}_{h}, q_{h}\right)-\tau\left(\operatorname{div} \boldsymbol{w}_{h}, q_{h}\right) .
\end{gathered}
$$

We then have the following theorem which shows that on every time step the discrete problem is solvable.

Theorem 3.2. If the triple $\left(\boldsymbol{V}_{h}, \boldsymbol{W}_{h}, Q_{h}\right)$ is Stokes-Biot stable, then:

$B(\cdot, \cdot, \cdot ; \cdot, \cdot, \cdot)$ is continuous with respect to $\|(\cdot, \cdot, \cdot)\| \| ;$ and

the following inf-sup condition holds.

$$
\sup _{\left(\boldsymbol{v}_{h}, \boldsymbol{r}_{h}, q_{h}\right) \in \boldsymbol{V}_{h} \times \boldsymbol{W}_{h} \times Q_{h}} \frac{B\left(\boldsymbol{u}_{h}, \boldsymbol{w}_{h}, p_{h} ; \boldsymbol{v}_{h}, \boldsymbol{r}_{h}, q_{h}\right)}{\left\|\left(\boldsymbol{u}_{h}, \boldsymbol{w}_{h}, p_{h}\right)\right\|} \geq \gamma\left\|\left(\boldsymbol{v}_{h}, \boldsymbol{r}_{h}, q_{h}\right)\right\|,
$$

with a constant $\gamma>0$ independent of mesh size $h$, time step size $\tau$, and the physical parameters.

Proof. The proof of Theorem 3.2 follows directly from Case I in the proof found in [16, Theorem 6].

Remark 3.3. The weighted norm, (24), used here is slightly different from the norm used in [16]. In [16], the authors are concerned with the tightness of the bounds for the stability analysis. This is not the focus here, but nevertheless, (24) also provides parameter-robustness, while still involving the bulk modulus $\lambda+2 \mu / d$. This gives the norm some physical meaning and has been shown to be effective in practice for other iterative schemes such as the fixed-stress splitting scheme [20].

Note that if we replace $a(\cdot, \cdot)$ with any spectrally equivalent bilinear form on $\boldsymbol{V}_{h} \times \boldsymbol{V}_{h}$, the same stability result holds true. In the next section, we introduce such a spectrally equivalent bilinear form which allows for: (1) Efficient elimination of the degrees of freedom corresponding to the bubble functions via static condensation; and (2) Derivation of optimal error estimates for the fully discrete problem, following the analysis in [33].

\section{Local perturbation of the bilinear form and elimination of bubbles}

A straightforward elimination of the edge/face bubbles is not local, and, in general, leads to a prohibitively large number of non-zeros in the resulting linear system. To resolve this, we introduce a consistent perturbation of $a(\cdot, \cdot)$, which has a diagonal matrix representation. It is then easy to eliminate the unknowns corresponding to the bubble functions in $\boldsymbol{V}_{b}$ with no fill-in. This leads to a stable P1-RT0-P0 discretization for the Biot's model and, consequently, to a stable P1-P0 discretization for the Stokes' equation.

First, consider a natural decomposition of $\boldsymbol{u} \in \boldsymbol{V}_{h}$ :

$$
\boldsymbol{u}=\boldsymbol{u}_{l}+\boldsymbol{u}_{b}=\underbrace{\Pi_{1} \boldsymbol{u}}_{\boldsymbol{u}_{l}}+\underbrace{\sum_{e \in \mathcal{E}^{o, t}} u_{e} \boldsymbol{\Phi}_{e}}_{\boldsymbol{u}_{b}}
$$

and the local bilinear forms for $T \in \mathcal{T}_{h}, \boldsymbol{u} \in \boldsymbol{V}_{h}$, and $\boldsymbol{v} \in \boldsymbol{V}_{h}$ :

$$
a_{T}(\boldsymbol{u}, \boldsymbol{v})=2 \mu \int_{T} \varepsilon(\boldsymbol{u}): \varepsilon(\boldsymbol{v})+\lambda \int_{T} \operatorname{div} \boldsymbol{u} \operatorname{div} \boldsymbol{v} .
$$

For the restriction of $a(\cdot, \cdot)$ onto the space spanned by bubble functions $\boldsymbol{V}_{b}$, we have

$$
a_{b}\left(\boldsymbol{u}_{b}, \boldsymbol{v}_{b}\right):=a\left(\boldsymbol{u}_{b}, \boldsymbol{v}_{b}\right)=\sum_{T \in \mathcal{T}_{h}} a_{b, T}\left(\boldsymbol{u}_{b}, \boldsymbol{v}_{b}\right)=\sum_{T \in \mathcal{T}_{h}} \sum_{e, e^{\prime} \in \partial T} u_{e} v_{e^{\prime}} a_{T}\left(\boldsymbol{\Phi}_{e^{\prime}}, \boldsymbol{\Phi}_{e}\right) .
$$

On each element, $T \in \mathcal{T}_{h}$, then introduce

$$
d_{b, T}(\boldsymbol{u}, \boldsymbol{v})=(d+1) \sum_{e \in \partial T} u_{e} v_{e} a_{T}\left(\boldsymbol{\Phi}_{e}, \boldsymbol{\Phi}_{e}\right), \quad d_{b}(\boldsymbol{u}, \boldsymbol{v})=\sum_{T \in \mathcal{T}_{h}} d_{b, T}(\boldsymbol{u}, \boldsymbol{v}) .
$$

Replacing $a_{b}(\cdot, \cdot)$ with $d_{b}(\cdot, \cdot)$ gives a perturbation, $a^{D}(\cdot, \cdot)$, of $a(\cdot, \cdot)$ :

$$
a^{D}(\boldsymbol{u}, \boldsymbol{v}):=d_{b}\left(\boldsymbol{u}_{b}, \boldsymbol{v}_{b}\right)+a\left(\boldsymbol{u}_{b}, \boldsymbol{v}_{l}\right)+a\left(\boldsymbol{u}_{l}, \boldsymbol{v}_{b}\right)+a\left(\boldsymbol{u}_{l}, \boldsymbol{v}_{l}\right) .
$$




\subsection{A spectral equivalence result}

To prove that the form $a^{D}(\cdot, \cdot)$ and $a(\cdot, \cdot)$ are spectrally equivalent, we need several auxiliary results. First, recall the definition of the rigid body motions (modes), $\mathfrak{R}$ on $\mathbb{R}^{d}$ :

$$
\mathfrak{R}=\left\{\boldsymbol{v}=\boldsymbol{a}+\mathfrak{b} \boldsymbol{x} \mid \boldsymbol{a} \in \mathbb{R}^{d}, \quad \mathfrak{b} \in \mathfrak{s o}(d)\right\},
$$

where $\mathfrak{s o}(d)$ is the algebra of skew-symmetric $(d \times d)$ matrices. The dimension of $\Re$ is $\frac{1}{2} d(d+1)$ and its elements are component-wise linear vector-valued functions.

Next, recall the classical Korn inequality [34,35] for $\boldsymbol{u} \in \boldsymbol{H}^{1}(Y)$ for a domain $Y \subset \mathbb{R}^{d}$, star-shaped with respect to a ball. As shown by Kondratiev and Oleinik in [36,37],

$$
\inf _{\mathfrak{m} \in \mathfrak{s o}(d)}\|\nabla \boldsymbol{u}-\mathfrak{m}\|_{L^{2}(Y)} \lesssim\|\varepsilon(\boldsymbol{u})\|_{L^{2}(Y)},
$$

where the constant hidden in $\lesssim$ depends on the shape regularity of $Y$, that is, on the ratio $\frac{\operatorname{diam}(Y)}{R}$. For convenience when referencing (30) later, we state the following lemma, which gives a simpler version of the inequality defined on simplices, where $Y=T \in \mathcal{T}_{h}$.

Lemma 4.1. Let $\mathcal{T}_{h}$ be a shape-regular simplicial mesh covering $\Omega$. Then, the following inequality holds for any $T \in \mathcal{T}_{h}$ and $\boldsymbol{u} \in \boldsymbol{H}^{1}(T)$ :

$$
\inf _{\mathfrak{m} \in \mathfrak{s o}(n)}\|\nabla \boldsymbol{u}-\mathfrak{m}\|_{L^{2}(T)} \lesssim\|\varepsilon(\boldsymbol{u})\|_{L^{2}(T)},
$$

where the constant hidden in "§” depends on the shape regularity constant of $\mathcal{T}_{h}$.

Defining the unscaled bilinear form, $\widetilde{d}_{b, T}$,

$$
\tilde{d}_{b, T}(\boldsymbol{u}, \boldsymbol{v}):=\sum_{e \in \partial T} u_{e} v_{e} a_{T}\left(\boldsymbol{\Phi}_{e}, \boldsymbol{\Phi}_{e}\right),
$$

we have the following local, spectral equivalence result.

Lemma 4.2. For all $T \in \mathcal{T}_{h}$ the following inequalities hold:

$$
\eta_{T} \widetilde{d}_{b, T}(\boldsymbol{u}, \boldsymbol{u}) \leq a_{b, T}(\boldsymbol{u}, \boldsymbol{u}) \leq(d+1) \widetilde{d}_{b, T}(\boldsymbol{u}, \boldsymbol{u}), \quad \text { for all } \boldsymbol{u} \in \boldsymbol{V}_{b},
$$

where the constant $\eta_{T}$ is independent of $h_{T}$ and $\rho_{T}$.

Proof. Set $a_{e e^{\prime}}=a_{b, T}\left(\boldsymbol{\Phi}_{e^{\prime}}, \boldsymbol{\Phi}_{e}\right)$ and note that $a_{e e}=\widetilde{d}_{b, T}\left(\boldsymbol{\Phi}_{e}, \boldsymbol{\Phi}_{e}\right)$ for all $e, e^{\prime} \in \partial T$. The upper bound follows immediately by two applications of the Cauchy-Schwarz inequality:

$$
\begin{aligned}
a_{b, T}(\boldsymbol{u}, \boldsymbol{u}) & =\sum_{e, e^{\prime} \in \partial T} a_{e e^{\prime}} u_{e} u_{e^{\prime}} \leq \sum_{e, e^{\prime} \in \partial T} \sqrt{a_{e e} a_{e^{\prime} e^{\prime}}}\left|u_{e} u_{e^{\prime}}\right|=\left(\sum_{e \in \partial T} \sqrt{a_{e e}}\left|u_{e}\right|\right)^{2} \\
& \leq(d+1) \sum_{e \in \partial T} a_{e e} u_{e}^{2}=(d+1) \tilde{d}_{b, T}(\boldsymbol{u}, \boldsymbol{u}) .
\end{aligned}
$$

We prove the lower bound by establishing the following inequalities for $\boldsymbol{u} \in \boldsymbol{V}_{b}$ :

$$
h_{T}^{-2}\|\boldsymbol{u}\|_{L^{2}(T)}^{2} \lesssim a_{b, T}(\boldsymbol{u}, \boldsymbol{u}), \quad \text { and } \quad \tilde{d}_{b, T}(\boldsymbol{u}, \boldsymbol{u}) \lesssim h_{T}^{-2}\|\boldsymbol{u}\|_{L^{2}(T)}^{2} .
$$

By definition for all $\boldsymbol{u} \in \boldsymbol{V}_{b}$ and all rigid body modes $\mathfrak{r} \in \mathfrak{R}$, we have that $\Pi_{1} \boldsymbol{u}=\mathbf{0}$ and $\Pi_{1} \mathfrak{r}=\mathfrak{r}$. The classical interpolation estimates found in [38, Chapter 3] give

$$
\|\boldsymbol{u}\|_{L^{2}(T)}^{2}=\left\|\boldsymbol{u}-\mathfrak{r}-\Pi_{1}(\boldsymbol{u}-\mathfrak{r})\right\|_{L^{2}(T)}^{2} \lesssim h_{T}^{2}\|\nabla(\boldsymbol{u}-\mathfrak{r})\|_{L^{2}(T)}^{2} .
$$

Taking the infimum over all $\mathfrak{r} \in \mathfrak{R}$ and applying Korn's inequality (Lemma 4.1) then yields

$$
h_{T}^{-2}\|\boldsymbol{u}\|_{L^{2}(T)}^{2} \lesssim \inf _{\mathfrak{r} \in \mathfrak{R}}\|\nabla(\boldsymbol{u}-\mathfrak{r})\|_{L^{2}(T)}^{2}=\inf _{\mathfrak{m} \in \mathfrak{s} \mathfrak{o}(d)}\|\nabla \boldsymbol{u}-\mathfrak{m}\|_{L^{2}(T)}^{2} \lesssim\|\varepsilon(\boldsymbol{u})\|_{L^{2}(T)}^{2} .
$$


This shows the first inequality in (34), and to prove the second inequality, we note that from the definition of $\widetilde{d}_{b, T}(\cdot, \cdot)$ and the inverse inequality, we have that

$$
\widetilde{d}_{b, T}(\boldsymbol{u}, \boldsymbol{u}) \lesssim \sum_{e \in \partial T} u_{e}^{2}\left[\left\|\nabla \boldsymbol{\Phi}_{e}\right\|_{L^{2}(T)}^{2}+\lambda\left\|\operatorname{div} \boldsymbol{\Phi}_{e}\right\|_{L^{2}(T)}^{2}\right] \lesssim h_{T}^{-2} \sum_{e \in \partial T} u_{e}^{2}\left\|\boldsymbol{\Phi}_{e}\right\|_{L^{2}(T)}^{2}
$$

Recalling the definition of $\Phi_{e}$ in (21) and the formula for integrating powers of the barycentric coordinates, gives

$$
\boldsymbol{\Phi}_{e}=\varphi_{e} \boldsymbol{n}_{e}, \quad \int_{T} \lambda_{1}^{\beta_{1}} \ldots \lambda_{d+1}^{\beta_{d+1}} d x=|T| \frac{\beta_{1} ! \ldots \beta_{d+1} ! d !}{\left(\beta_{1}+\cdots+\beta_{d+1}+d\right) !} .
$$

It follows that $\left\|\boldsymbol{\Phi}_{e}\right\|_{L^{2}(T)}^{2}=c_{d}|T|$ and $\int_{T} \boldsymbol{\Phi}_{e} \boldsymbol{\Phi}_{e^{\prime}}=\frac{1}{2} c_{d}|T|\left(\delta_{e e^{\prime}}+\boldsymbol{n}_{e} \cdot \boldsymbol{n}_{e^{\prime}}\right)$, with $c_{d}=\frac{d ! 2^{d}}{(3 d) !}$. As the Gram matrix $\left(\boldsymbol{n}_{e} \cdot \boldsymbol{n}_{e^{\prime}}\right)_{e, e^{\prime} \in \partial T}$ is positive semi-definite,

$$
\begin{aligned}
\sum_{e \in \partial T} u_{e}^{2}\left\|\boldsymbol{\Phi}_{e}\right\|_{L^{2}(T)}^{2} & =c_{d}|T| \sum_{e \in \partial T} u_{e}^{2} \leq c_{d}|T|\left[\sum_{e \in \partial T} u_{e}^{2}+\sum_{e, e^{\prime} \in \partial T} u_{e} u_{e^{\prime}}\left(\boldsymbol{n}_{e} \cdot \boldsymbol{n}_{e^{\prime}}\right)\right] \\
& =\left\|\sum_{e \in \partial T} u_{e} \boldsymbol{\Phi}_{e}\right\|_{L^{2}(T)}^{2}=\|\boldsymbol{u}\|_{L^{2}(T)}^{2} .
\end{aligned}
$$

Multiplying by $h_{T}^{-2}$ on both sides of this inequality furnishes the proof of (34), completing the proof of the lemma.

Next, we show the spectral equivalence for the bilinear forms $a(\cdot, \cdot)$ and $a^{D}(\cdot, \cdot)$.

\section{Lemma 4.3. The following inequalities hold:}

$$
a(\boldsymbol{u}, \boldsymbol{u}) \leq a^{D}(\boldsymbol{u}, \boldsymbol{u}) \leq \eta a(\boldsymbol{u}, \boldsymbol{u}), \quad \text { for all } \boldsymbol{u} \in \boldsymbol{V}_{h},
$$

where $\eta$ depends on the shape regularity of the mesh.

Proof. Let $\boldsymbol{u} \in \boldsymbol{V}_{h}, \boldsymbol{u}=\boldsymbol{u}_{l}+\boldsymbol{u}_{b}$. From the definition of $d_{b}(\cdot, \cdot)$ in (28), $a_{b, T}\left(\boldsymbol{u}_{b}, \boldsymbol{u}_{b}\right) \leq d_{b, T}\left(\boldsymbol{u}_{b}, \boldsymbol{u}_{b}\right)$, and the lower bound follows immediately:

$$
a(\boldsymbol{u}, \boldsymbol{u})-a^{D}(\boldsymbol{u}, \boldsymbol{u})=a_{b}\left(\boldsymbol{u}_{b}, \boldsymbol{u}_{b}\right)-d_{b}\left(\boldsymbol{u}_{b}, \boldsymbol{u}_{b}\right)=\sum_{T \in \mathcal{T}_{h}}\left[a_{b, T}\left(\boldsymbol{u}_{b}, \boldsymbol{u}_{b}\right)-d_{b, T}\left(\boldsymbol{u}_{b}, \boldsymbol{u}_{b}\right)\right] \leq 0 .
$$

To prove the upper bound, we use the following local estimate, which is established using an inverse inequality, a standard interpolation estimate, and $\Pi_{1} \mathfrak{r}=\mathfrak{r}$ for all rigid body modes $\mathfrak{r} \in \mathfrak{R}$,

$$
\begin{aligned}
a_{T}\left(\boldsymbol{u}_{b}, \boldsymbol{u}_{b}\right) & \lesssim\left\|\nabla \boldsymbol{u}_{b}\right\|_{L^{2}(T)}^{2} \lesssim h_{T}^{-2}\left\|\boldsymbol{u}_{b}\right\|_{L^{2}(T)}^{2}=h_{T}^{-2}\left\|\boldsymbol{u}-\Pi_{1} \boldsymbol{u}\right\|_{L^{2}(T)}^{2} \\
& =h_{T}^{-2}\left\|(\boldsymbol{u}-\mathfrak{r})-\Pi_{1}(\boldsymbol{u}-\mathfrak{r})\right\|_{L^{2}(T)}^{2} \lesssim\|\nabla(\boldsymbol{u}-\mathfrak{r})\|_{L^{2}(T)}^{2} .
\end{aligned}
$$

Taking the infimum over all $\mathfrak{r} \in \mathfrak{R}$ and applying the Korn's inequality (Lemma 4.1) then yields

$$
a_{T}\left(\boldsymbol{u}_{b}, \boldsymbol{u}_{b}\right) \lesssim \inf _{\mathfrak{r} \in \mathfrak{R}}\|\nabla(\boldsymbol{u}-\mathfrak{r})\|_{L^{2}(T)}^{2}=\inf _{\mathfrak{m} \in \mathfrak{s o}(d)}\|\nabla \boldsymbol{u}-\mathfrak{m}\|_{L^{2}(T)}^{2} \lesssim\|\varepsilon(\boldsymbol{u})\|_{L^{2}(T)}^{2} .
$$

This inequality, combined with the definition of $a^{D}(\cdot, \cdot)$, and the lower bound in Lemma 4.2 gives,

$$
\begin{aligned}
a^{D}(\boldsymbol{u}, \boldsymbol{u}) & =a(\boldsymbol{u}, \boldsymbol{u})+\sum_{T \in \mathcal{T}_{h}} d_{b, T}\left(\boldsymbol{u}_{b}, \boldsymbol{u}_{b}\right)-a_{T}\left(\boldsymbol{u}_{b}, \boldsymbol{u}_{b}\right) \\
& \leq a(\boldsymbol{u}, \boldsymbol{u})+\sum_{T \in \mathcal{T}_{h}}\left(\frac{d+1}{\eta_{T}}-1\right) a_{T}\left(\boldsymbol{u}_{b}, \boldsymbol{u}_{b}\right) \\
& \lesssim a(\boldsymbol{u}, \boldsymbol{u})+\sum_{T \in \mathcal{T}_{h}}\left(\frac{d+1}{\eta_{T}}-1\right)\|\varepsilon(\boldsymbol{u})\|_{L^{2}(T)}^{2} \\
& \lesssim a(\boldsymbol{u}, \boldsymbol{u}) . \quad \square
\end{aligned}
$$


Since we have shown that the bilinear form $a^{D}(\cdot, \cdot)$ can replace $a(\cdot, \cdot)$ in Definition 3.1, then Theorem 3.2 holds when the bilinear form, $B(\cdot, \cdot, \cdot ; \cdot, \cdot, \cdot)$, has $a^{D}(\cdot, \cdot)$ instead of $a(\cdot, \cdot)$. Thus, the variational problem,

$$
\begin{aligned}
& a^{D}\left(\boldsymbol{u}_{h}^{m}, \boldsymbol{v}_{h}\right)-\left(\alpha p_{h}^{m}, \operatorname{div} \boldsymbol{v}_{h}\right)=\left(\rho \boldsymbol{g}, \boldsymbol{v}_{h}\right), \quad \forall \boldsymbol{v}_{h} \in \boldsymbol{V}_{h}, \\
& \left(K^{-1} \mu_{f} \boldsymbol{w}_{h}^{m}, \boldsymbol{r}_{h}\right)-\left(p_{h}^{m}, \operatorname{div} \boldsymbol{r}_{h}\right)=\left(\rho_{f} \boldsymbol{g}, \boldsymbol{r}_{h}\right), \quad \forall \boldsymbol{r}_{h} \in \boldsymbol{W}_{h}, \\
& \left(\frac{1}{M} \bar{\partial}_{t} p_{h}^{m}, q_{h}\right)+\left(\alpha \operatorname{div} \bar{\partial}_{t} \boldsymbol{u}_{h}^{m}, q_{h}\right)+\left(\operatorname{div} \boldsymbol{w}_{h}^{m}, q_{h}\right)=\left(f, q_{h}\right), \quad \forall q_{h} \in Q_{h},
\end{aligned}
$$

has a unique solution and defines an invertible operator with inverse bounded independent of the mesh size $h$. This observation plays a crucial role in the error estimates in the next subsection.

For later comparison, we define following block form of the discrete problem:

$$
\mathcal{A}^{D}\left(\begin{array}{l}
\boldsymbol{U}_{b} \\
\boldsymbol{U}_{l} \\
\boldsymbol{W} \\
\boldsymbol{P}
\end{array}\right)=\boldsymbol{b} \text {, with } \mathcal{A}^{D}=\left(\begin{array}{cccc}
D_{b b} & A_{b l} & 0 & G_{b} \\
A_{b l}^{T} & A_{l l} & 0 & G_{l} \\
0 & 0 & \tau M_{w} & \tau G \\
G_{b}^{T} & G_{l}^{T} & \tau G^{T} & -M_{p}
\end{array}\right),
$$

where everything is defined as before and $D_{b b}$ corresponds to $a^{D}\left(\boldsymbol{u}_{h}^{b}, \boldsymbol{v}_{h}^{b}\right)$.

\subsection{Error estimates for the fully discrete problem}

To derive the error analysis of the fully discrete scheme, following the standard error analysis of time-dependent problems in Thomée [39], we first define the following elliptic projections $\overline{\boldsymbol{u}}_{h} \in \boldsymbol{V}_{h}, \overline{\boldsymbol{w}}_{h} \in \boldsymbol{W}_{h}$, and $\bar{p}_{h} \in Q_{h}$ for $t>0$ as usual,

$$
\begin{aligned}
& a^{D}\left(\overline{\boldsymbol{u}}_{h}, \boldsymbol{v}_{h}\right)-\left(\alpha \bar{p}_{h}, \operatorname{div} \boldsymbol{v}_{h}\right)=a\left(\boldsymbol{u}, \boldsymbol{v}_{h}\right)-\left(\alpha p, \operatorname{div} \boldsymbol{v}_{h}\right), \quad \forall \boldsymbol{v}_{h} \in \boldsymbol{V}_{h}, \\
& \left(K^{-1} \mu_{f} \overline{\boldsymbol{w}}_{h}, \boldsymbol{r}_{h}\right)-\left(\bar{p}_{h}, \operatorname{div} \boldsymbol{r}_{h}\right)=\left(K^{-1} \mu_{f} \boldsymbol{w}, \boldsymbol{r}_{h}\right)-\left(p, \operatorname{div} \boldsymbol{r}_{h}\right), \quad \forall \boldsymbol{r}_{h} \in \boldsymbol{W}_{h}, \\
& \left(\operatorname{div} \overline{\boldsymbol{w}}_{h}, q_{h}\right)=\left(\operatorname{div} \boldsymbol{w}, q_{h}\right), \quad \forall q_{h} \in Q_{h} .
\end{aligned}
$$

Note that the above elliptic projections are decoupled; $\overline{\boldsymbol{w}}_{h}$ and $\bar{p}_{h}$ are defined by (41) and (42), which is a mixed formulation of the Poisson equation. Therefore, the existence and uniqueness of $\overline{\boldsymbol{w}}_{h}$ and $\bar{p}_{h}$ follow directly from standard results. After $\bar{p}_{h}$ is defined, $\overline{\boldsymbol{u}}_{h}$ is then determined by solving (40), which is a linear elasticity problem, and again the existence and uniqueness of $\overline{\boldsymbol{u}}_{h}$ follow from standard results. Now, we split the errors as follows,

$$
\begin{aligned}
& \boldsymbol{u}\left(t_{n}\right)-\boldsymbol{u}_{h}^{n}=\left(\boldsymbol{u}\left(t_{n}\right)-\overline{\boldsymbol{u}}_{h}\left(t_{n}\right)\right)-\left(\boldsymbol{u}_{h}^{n}-\overline{\boldsymbol{u}}_{h}\left(t_{n}\right)\right)=: \rho_{\boldsymbol{u}}^{n}-e_{u}^{n}, \\
& \boldsymbol{w}\left(t_{n}\right)-\boldsymbol{w}_{h}^{n}=\left(\boldsymbol{w}\left(t_{n}\right)-\overline{\boldsymbol{w}}_{h}\left(t_{n}\right)\right)-\left(\boldsymbol{w}_{h}^{n}-\overline{\boldsymbol{w}}_{h}\left(t_{n}\right)\right)=: \rho_{\boldsymbol{w}}^{n}-e_{\boldsymbol{w}}^{n}, \\
& p\left(t_{n}\right)-p_{h}^{n}=\left(p\left(t_{n}\right)-\bar{p}_{h}\left(t_{n}\right)\right)-\left(p_{h}^{n}-\bar{p}_{h}\left(t_{n}\right)\right)=: \rho_{p}^{n}-e_{p}^{n} .
\end{aligned}
$$

Lemma 4.4. The following error estimates for the elliptic projections defined in (40)-(42) hold for $t>0$,

$$
\begin{aligned}
& \left\|\rho_{\boldsymbol{u}}\right\|_{1} \leq \operatorname{ch}\left(\|\boldsymbol{u}\|_{2}+\|p\|_{1}\right), \\
& \left\|\rho_{\boldsymbol{w}}\right\| \leq \operatorname{ch}\|\boldsymbol{w}\|_{1}, \\
& \left\|\rho_{p}\right\| \leq \operatorname{ch}\left(\|p\|_{1}+\|\boldsymbol{w}\|_{1}\right) .
\end{aligned}
$$

Proof. Error estimates in (44) and (45) follow from the error analysis of the mixed formulation of Poisson problems. The estimate (43) follows from the triangle inequality:

$$
\left\|\rho_{\boldsymbol{u}}\right\|_{1} \leq\left\|\boldsymbol{u}-\Pi_{1} \boldsymbol{u}\right\|_{1}+\left\|\Pi_{1} \boldsymbol{u}-\overline{\boldsymbol{u}}_{h}\right\|_{1},
$$

where $\Pi_{1} \boldsymbol{u}$ is the linear interpolant of $\boldsymbol{u}$. Using the coercivity of $a^{D}(\cdot, \cdot)$ and that $a^{D}\left(\Pi_{1} \boldsymbol{u}, \boldsymbol{v}_{h}\right)=a\left(\Pi_{1} \boldsymbol{u}, \boldsymbol{v}_{h}\right) \forall \boldsymbol{v}_{h} \in \boldsymbol{V}_{h}$, for the linear function, $\Pi_{1} \boldsymbol{u}$, we get,

$$
\alpha_{V}^{D}\left\|\Pi_{1} \boldsymbol{u}-\overline{\boldsymbol{u}}_{h}\right\|_{1}^{2} \leq a^{D}\left(\Pi_{1} \boldsymbol{u}-\overline{\boldsymbol{u}}_{h}, \Pi_{1} \boldsymbol{u}-\overline{\boldsymbol{u}}_{h}\right)=a\left(\Pi_{1} \boldsymbol{u}, \Pi_{1} \boldsymbol{u}-\overline{\boldsymbol{u}}_{h}\right)-a^{D}\left(\overline{\boldsymbol{u}}_{h}, \Pi_{1} \boldsymbol{u}-\overline{\boldsymbol{u}}_{h}\right) .
$$

Taking into account that $\overline{\boldsymbol{u}}_{h}$ is the solution of Eq. (40),

$$
\begin{aligned}
\alpha_{V}^{D}\left\|\Pi_{1} \boldsymbol{u}-\overline{\boldsymbol{u}}_{h}\right\|_{1}^{2} & \leq a\left(\Pi_{1} \boldsymbol{u}-\boldsymbol{u}, \Pi_{1} \boldsymbol{u}-\overline{\boldsymbol{u}}_{h}\right)+\alpha\left(p-\bar{p}_{h}, \operatorname{div}\left(\Pi_{1} \boldsymbol{u}-\overline{\boldsymbol{u}}_{h}\right)\right) \\
& \leq C_{\boldsymbol{V}}^{D}\left\|\Pi_{1} \boldsymbol{u}-\boldsymbol{u}\right\|_{1}\left\|\Pi_{1} \boldsymbol{u}-\overline{\boldsymbol{u}}_{h}\right\|_{1}+\alpha\left\|p-\bar{p}_{h}\right\|\left\|\Pi_{1} \boldsymbol{u}-\overline{\boldsymbol{u}}_{h}\right\|_{1} .
\end{aligned}
$$


Then, it follows that

$$
\left\|\rho_{\boldsymbol{u}}\right\|_{1} \leq\left(1+\frac{C_{\boldsymbol{V}}^{D}}{\alpha_{\boldsymbol{V}}^{D}}\right)\left\|\boldsymbol{u}-\Pi_{1} \boldsymbol{u}\right\|_{1}+\frac{\alpha}{\alpha_{V}^{D}}\left\|\rho_{p}\right\|
$$

The error estimate in (43) is obtained by using (45) and the standard error estimates for linear finite elements.

We similarly define the elliptic projection, $\overline{\partial_{t} \boldsymbol{u}_{h}}, \overline{\partial_{t} \boldsymbol{w}_{h}}$, and $\overline{\partial_{t} p_{h}}$ of $\partial_{t} \boldsymbol{u}_{h}, \partial_{t} \boldsymbol{w}_{h}$, and $\partial_{t} p_{h}$ respectively. This gives similar estimates as above for $\partial_{t} \rho_{\boldsymbol{u}}, \partial_{t} \rho_{\boldsymbol{w}}$, and $\partial_{t} \rho_{p}$, where on the right-hand side of the inequalities we use norms of $\partial_{t} \boldsymbol{u}_{h}, \partial_{t} \boldsymbol{w}_{h}$, and $\partial_{t} p_{h}$ instead of the norms of $\boldsymbol{u}_{h}, \boldsymbol{w}_{h}$, and $p_{h}$ respectively.

Next, we estimate the errors, $e_{u}, e_{w}$, and $e_{p}$ using the following norm,

$$
\left\|\left(\boldsymbol{u}_{h}, \boldsymbol{w}_{h}, p_{h}\right)\right\|_{\tau, h}:=\left(\left\|\boldsymbol{u}_{h}\right\|_{1}^{2}+\tau\left\|\boldsymbol{w}_{h}\right\|_{K^{-1} \mu_{f}}^{2}+\left(\frac{1}{M}+1\right)\left\|p_{h}\right\|^{2}\right)^{1 / 2},
$$

where $\left\|\boldsymbol{w}_{h}\right\|_{K^{-1} \mu_{f}}^{2}:=\left(K^{-1} \mu_{f} \boldsymbol{w}_{h}, \boldsymbol{w}_{h}\right)$.

Lemma 4.5. Let $R_{\boldsymbol{u}}^{j}:=\partial_{t} \boldsymbol{u}\left(t_{j}\right)-\frac{\overline{\boldsymbol{u}}_{h}\left(t_{j}\right)-\overline{\boldsymbol{u}}_{h}\left(t_{j-1}\right)}{\tau}$. Then,

$$
\left\|\left(e_{\boldsymbol{u}}^{m}, e_{\boldsymbol{w}}^{m}, e_{p}^{m}\right)\right\|_{\tau, h} \leq c\left(\left\|e_{\boldsymbol{u}}^{0}\right\|_{1}+\frac{1}{M}\left\|e_{p}^{0}\right\|+\tau \sum_{j=1}^{n}\left\|R_{\boldsymbol{u}}^{j}\right\|_{1}\right) .
$$

Proof. Choosing $\boldsymbol{v}=\boldsymbol{v}_{h}$ in (12), $\boldsymbol{r}=\boldsymbol{r}_{h}$ in (13), and $q=q_{h}$ in (14), subtracting these equations from (36)-(38), and using the definition of elliptic projections given in (40), (41), and (42) yields,

$$
\begin{aligned}
& a^{D}\left(e_{\boldsymbol{u}}^{m}, \boldsymbol{v}_{h}\right)-\left(\alpha e_{p}^{m}, \operatorname{div} \boldsymbol{v}_{h}\right)=0, \\
& \left(K^{-1} \mu_{f} e_{\boldsymbol{w}}^{m}, \boldsymbol{r}_{h}\right)_{h}-\left(e_{p}^{m}, \operatorname{div} \boldsymbol{r}_{h}\right)=0, \\
& -\left(\frac{1}{M} \bar{\partial}_{t} e_{p}^{m}, q_{h}\right)-\left(\alpha \operatorname{div} \bar{\partial}_{t} e_{\boldsymbol{u}}^{m}, q_{h}\right)-\left(\operatorname{div} e_{\boldsymbol{w}}^{m}, q_{h}\right)=-\left(\operatorname{div} R_{\boldsymbol{u}}^{m}, q_{h}\right) .
\end{aligned}
$$

Then, choosing $\boldsymbol{v}_{h}=\bar{\partial}_{t} e_{\boldsymbol{u}}^{m}, \boldsymbol{r}_{h}=e_{\boldsymbol{w}}^{m}$ and $q_{h}=-e_{p}^{m}$ in (47), (48), and (49), respectively, and adding these equations, yields,

$$
\left\|e_{u}^{m}\right\|_{a^{D}}^{2}+\tau\left\|e_{w}^{m}\right\|_{K^{-1} \mu_{f}}^{2}+\frac{1}{M}\left\|e_{p}^{m}\right\|^{2} \leq\left\|e_{u}^{m}\right\|_{a^{D}}\left\|e_{u}^{m-1}\right\|_{a^{D}}+\frac{1}{M}\left\|e_{p}^{m}\right\|\left\|e_{p}^{m-1}\right\|+\tau\left\|\operatorname{div} R_{u}^{m}\right\|\left\|e_{p}^{m}\right\| .
$$

Using the inf-sup condition corresponding to the mixed formulation of the Darcy problem with RT0-P0 and using the equality in (48) gives,

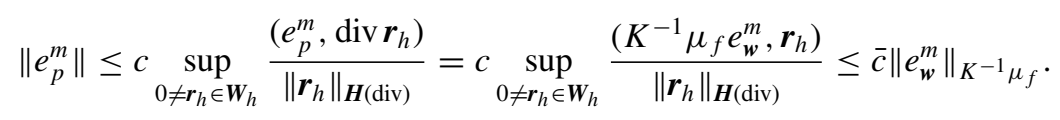

By applying $a b \leq \frac{a^{2}}{2}+\frac{b^{2}}{2}$ and the bound in (50), the following inequality holds,

$$
\left\|e_{\boldsymbol{u}}^{m}\right\|_{a^{D}}^{2}+\tau\left\|e_{\boldsymbol{w}}^{m}\right\|_{K^{-1} \mu_{f}}^{2}+\frac{1}{M}\left\|e_{p}^{m}\right\|^{2} \leq\left\|e_{\boldsymbol{u}}^{m-1}\right\|_{a^{D}}^{2}+\frac{1}{M}\left\|e_{p}^{m-1}\right\|^{2}+c \tau\left\|R_{\boldsymbol{u}}^{m}\right\|_{1}^{2} .
$$

This implies by recursion that

$$
\left\|e_{\boldsymbol{u}}^{m}\right\|_{a^{D}}^{2}+\tau\left\|e_{\boldsymbol{w}}^{m}\right\|_{K^{-1} \mu_{f}}^{2}+\frac{1}{M}\left\|e_{p}^{m}\right\|^{2} \leq\left\|e_{u}^{0}\right\|_{a^{D}}^{2}+\frac{1}{M}\left\|e_{p}^{0}\right\|^{2}+c \tau \sum_{j=1}^{m}\left\|R_{u}^{j}\right\|_{1}^{2} .
$$

From the coercivity and continuity of the bilinear form, $a^{D}(\cdot, \cdot)$, the estimate in (46) is obtained.

Finally, following the same procedures of Lemma 8 in [14], we have

$$
\sum_{j=1}^{n}\left\|R_{\boldsymbol{u}}^{j}\right\|_{1} \leq c\left(\int_{0}^{t_{n}}\left\|\partial_{t t} \boldsymbol{u}\right\|_{1} \mathrm{~d} t+\frac{1}{\tau} \int_{0}^{t_{n}}\left\|\partial_{t} \rho_{\boldsymbol{u}}\right\|_{1} \mathrm{~d} t\right) .
$$

Thus, we derive the following error estimates. 
Theorem 4.6. Let $\boldsymbol{u}, \boldsymbol{w}$, and $p$ be the solutions of (12)-(14) and $\boldsymbol{u}_{h}^{n}$, $\boldsymbol{w}_{h}^{n}$, and $p_{h}^{n}$ be the solutions of (36)-(38). If the following regularity assumptions hold,

$$
\begin{aligned}
& \boldsymbol{u}(t) \in L^{\infty}\left((0, T], \mathbf{H}_{0}^{1}(\Omega)\right) \cap L^{\infty}\left((0, T], \mathbf{H}^{2}(\Omega)\right), \\
& \partial_{t} \boldsymbol{u} \in L^{1}\left((0, T], \mathbf{H}^{2}(\Omega)\right), \partial_{t t} \boldsymbol{u} \in L^{1}\left((0, T], \mathbf{H}^{1}(\Omega)\right), \\
& \boldsymbol{w}(t) \in L^{\infty}\left((0, T], H_{0}(\operatorname{div}, \Omega)\right) \cap L^{\infty}\left((0, T], \mathbf{H}^{1}(\Omega)\right), \\
& p \in L^{\infty}\left((0, T], H^{1}(\Omega)\right), \partial_{t} p \in L^{1}\left((0, T], H^{1}(\Omega)\right),
\end{aligned}
$$

then,

$$
\begin{gathered}
\left\|\left(\boldsymbol{u}\left(t_{n}\right)-\boldsymbol{u}_{h}^{n}, \boldsymbol{w}\left(t_{n}\right)-\boldsymbol{w}_{h}^{n}, p\left(t_{n}\right)-p_{h}^{n}\right)\right\|_{\tau, h} \leq c\left\{\left\|e_{u}^{0}\right\|_{1}+\frac{1}{M}\left\|e_{p}^{0}\right\|+\tau \int_{0}^{t_{n}}\left\|\partial_{t t} \boldsymbol{u}\right\|_{1} \mathrm{~d} t\right. \\
\left.+h\left[\|\boldsymbol{u}\|_{2}+\tau^{1 / 2}\|\boldsymbol{w}\|_{1}+\|\boldsymbol{w}\|_{1}+\|p\|_{1}+\int_{0}^{t_{n}}\left(\left\|\partial_{t} \boldsymbol{u}\right\|_{2}+\left\|\partial_{t} p\right\|_{1}\right) \mathrm{d} t\right]\right\} .
\end{gathered}
$$

Proof. The error estimate follows directly from (46), (51), (43)-(45), and the triangle inequality.

\subsection{Practical implementation}

Since $d_{b}(\cdot, \cdot)$ has a diagonal matrix representation, we can eliminate the degrees of freedom corresponding to the bubble functions in order to have the same degrees of freedom as in the original P1-RT0-P0 method for the three-field formulation of the poroelasticity problem. After eliminating such unknowns from (39), we obtain a $(3 \times 3)$ block discrete linear system with similar blocks:

$$
\widehat{\mathcal{A}}^{D}=\left(\begin{array}{ccc}
A_{l l}-A_{b l}^{T} D_{b b}^{-1} A_{b l} & 0 & G_{l}-A_{b l}^{T} D_{b b}^{-1} G_{b} \\
0 & \tau M_{w} & \tau G \\
G_{l}^{T}-G_{b}^{T} D_{b b}^{-1} A_{b l} & \tau G^{T} & -M_{p}-G_{b}^{T} D_{b b}^{-1} G_{b}
\end{array}\right) .
$$

\section{Stabilized P1-P0 discretization for the Stokes problem}

When the permeability tends to zero in the poroelasticity problem, a Stokes-type problem is obtained. Thus, all the results obtained above can be directly applied to Stokes' equations. In particular, after the elimination of the bubble functions, one obtains a finite-element pair for the Stokes' system, based on piecewise linear finite elements for the velocity and piecewise constant functions for the pressure. This gives a Stokes-stable finite-element method with a minimum number of degrees of freedom.

To illustrate this further, consider the Stokes' problem for steady flow,

$$
\begin{aligned}
& -\operatorname{div}(2 v \boldsymbol{\varepsilon}(\boldsymbol{u})-p \boldsymbol{I})=\boldsymbol{f}, \text { in } \Omega, \\
& \operatorname{div} \boldsymbol{u}=0, \text { in } \Omega, \\
& \boldsymbol{u}=\mathbf{0}, \text { on } \Gamma,
\end{aligned}
$$

where $\boldsymbol{u}$ denotes the fluid velocity, $p$ is the pressure, $v$ is the viscosity constant, $\boldsymbol{f} \in\left(L^{2}(\Omega)\right)^{d}$ is a given forcing term acting on the fluid, and $\boldsymbol{\varepsilon}(\boldsymbol{u})=\frac{1}{2}\left(\nabla \boldsymbol{u}+\nabla \boldsymbol{u}^{t}\right)$. By considering $\boldsymbol{V}=\boldsymbol{H}_{0}^{1}(\Omega)=\left\{\boldsymbol{u} \in \boldsymbol{H}^{1}(\Omega) \mid \boldsymbol{u}=\mathbf{0}\right.$ on $\left.\Gamma\right\}$ and $Q=L_{0}^{2}(\Omega)=L^{2}(\Omega) / \mathbb{R}$ as the subspace of $L^{2}(\Omega)$ consisting of functions with zero mean value on $\Omega$, we write the weak formulation of problem (54)-(56) as follows

$$
\begin{aligned}
& a^{S}(\boldsymbol{u}, \boldsymbol{v})-(p, \operatorname{div} \boldsymbol{v})=(\boldsymbol{f}, \boldsymbol{v}), \quad \forall \boldsymbol{v} \in \boldsymbol{V}, \\
& (\operatorname{div} \boldsymbol{u}, q)=0, \quad \forall q \in Q,
\end{aligned}
$$

where $a^{S}(\boldsymbol{u}, \boldsymbol{v})=2 v \int_{\Omega} \boldsymbol{\varepsilon}(\boldsymbol{u}): \boldsymbol{\varepsilon}(\boldsymbol{v})$. As in the previous sections, we introduce the following finite-dimensional subspaces. For velocity, let $\boldsymbol{V}_{h}$ be the space of piecewise linear elements enriched with the normal components of face bubble functions. For pressure, let $Q_{h}$ be the subspace of piecewise constant functions. Then, the discrete variational formulation is given by: 
Find $\left(\boldsymbol{u}_{h}, p_{h}\right) \in \boldsymbol{V}_{h} \times Q_{h}$ such that

$$
\begin{aligned}
& a^{S}\left(\boldsymbol{u}_{h}, \boldsymbol{v}_{h}\right)-\left(p_{h}, \operatorname{div} \boldsymbol{v}_{h}\right)=\left(\boldsymbol{f}, \boldsymbol{v}_{h}\right), \quad \forall \boldsymbol{v}_{h} \in \boldsymbol{V}_{h}, \\
& \left(\operatorname{div} \boldsymbol{u}_{h}, q_{h}\right)=0 . \quad \forall q_{h} \in Q_{h},
\end{aligned}
$$

This formulation gives rise to the following block form of the fully discrete problem,

$$
\mathcal{A}_{S}\left(\begin{array}{c}
\boldsymbol{U}_{b} \\
\boldsymbol{U}_{l} \\
\boldsymbol{P}
\end{array}\right)=\boldsymbol{b} \text {, with } \mathcal{A}_{S}=\left(\begin{array}{ccc}
A_{b b} & A_{b l} & G_{b} \\
A_{b l}^{T} & A_{l l} & G_{l} \\
G_{b}^{T} & G_{l}^{T} & 0
\end{array}\right),
$$

where $\boldsymbol{U}_{b}, \boldsymbol{U}_{l}$, and $\boldsymbol{P}$ are the unknown vectors corresponding to the bubble component of the velocity, the linear component of the velocity, and the pressure, respectively. With the aim of eliminating the degrees of freedom corresponding to the bubble functions, we replace $A_{b b}$ by a spectrally-equivalent diagonal matrix $D_{b b}$, obtaining the following block form of the coefficient matrix,

$$
\mathcal{A}_{S}^{D}=\left(\begin{array}{ccc}
D_{b b} & A_{b l} & G_{b} \\
A_{b l}^{T} & A_{l l} & G_{l} \\
G_{b}^{T} & G_{l}^{T} & 0
\end{array}\right)
$$

Finally, we eliminate unknowns corresponding to the bubbles to obtain a 2 by 2 system,

$$
\widehat{\mathcal{A}}_{S}^{D}=\left(\begin{array}{cc}
A_{l l}-A_{b l}^{T} D_{b b}^{-1} A_{b l} & G_{l}-A_{b l}^{T} D_{b b}^{-1} G_{b} \\
G_{l}^{T}-G_{b}^{T} D_{b b}^{-1} A_{b l} & -G_{b}^{T} D_{b b}^{-1} G_{b}
\end{array}\right) .
$$

The resulting scheme is a stabilized $\mathrm{P} 1-\mathrm{P} 0$ discretization of Stokes in which stabilization terms appear in every subblock. Optimal order error estimates for this stabilized scheme follow from the estimates provided in [32, pp. 145-149] for the pair of spaces $\left(\boldsymbol{V}_{h}, Q_{h}\right), \boldsymbol{V}_{h}=\boldsymbol{V}_{h, 1} \oplus \boldsymbol{V}_{b}$.

\subsection{Mass conservation}

Finally, we briefly comment on an efficient post-processing step to ensure that the numerical solution obtained above preserves mass. Let $\left(\boldsymbol{u}_{h}, p_{h}\right) \in \boldsymbol{V}_{h} \times Q_{h}$, with $\boldsymbol{u}_{h}=\boldsymbol{u}_{l}+\boldsymbol{u}_{b}$ be the numerical solution to Stokes' equation obtained in the following way: first, we solve System (63) for $u_{l}$; and then, we compute $\boldsymbol{u}_{b}$. Note that the second step requires only the solution of systems with $D_{b b}$, which is a diagonal matrix. A mass-conserving approximation is then obtained by interpolating the numerical solution using the interpolant from the lowest-order BDM space (see Brezzi, Douglas and Marini [40], and Brezzi, Douglas, Duran and Fortin [41] for more details).

More specifically, let $\Pi_{h}^{\mathrm{BDM}}$ be the standard interpolation operator in the BDM space as defined in [29], [42, Section 5.4]. From the commuting diagram property of BDM elements (see, e.g. [31, Proposition 2.5.2]),

$$
\operatorname{div} \Pi_{h}^{\mathrm{BDM}} \boldsymbol{w}=\Pi_{h}^{0} \operatorname{div} \boldsymbol{w},
$$

for all sufficiently smooth $\boldsymbol{w} \in \boldsymbol{V}$. Here, $\Pi_{h}^{0}$ is the $L^{2}(\Omega)$-orthogonal projection on the space of piecewise constants, $Q_{h}$. This implies that

$$
\int_{\Omega} \operatorname{div} \Pi_{h}^{\mathrm{BDM}} \boldsymbol{u}_{h} q_{h}=\int_{\Omega} \operatorname{div} \boldsymbol{u}_{h} q_{h}=0, \quad \text { for all } q_{h} \in Q_{h},
$$

which shows that $\Pi_{h}^{\mathrm{BDM}} \boldsymbol{u}_{h}$ is indeed mass conservative.

Furthermore, we show that $\Pi_{h}^{\mathrm{BDM}} \boldsymbol{u}_{h}$ also approximates the solution, $\boldsymbol{u}$, to Stokes' equation in the $L^{2}(\Omega)$-norm. We recall the following classical error estimate for the BDM interpolant (see, e.g. [31, Proposition 2.5.4], [42, Theorem 5.25]):

$$
\left\|\boldsymbol{w}-\Pi_{h}^{\mathrm{BDM}} \boldsymbol{w}\right\| \lesssim h|\boldsymbol{w}|_{1} .
$$

As a consequence from (65),

$$
\left\|\Pi_{h}^{\mathrm{BDM}} \boldsymbol{w}\right\| \leq\left\|\boldsymbol{w}-\Pi_{h}^{\mathrm{BDM}} \boldsymbol{w}\right\|+\|\boldsymbol{w}\| \lesssim h|\boldsymbol{w}|_{1}+\|\boldsymbol{w}\| .
$$


Table 6.1

Energy norm for displacement errors and $L^{2}$-norm for pressure errors by considering different values of $\kappa$ and different mesh-sizes, using the "diagonal" bubble formulation, $\mathcal{A}^{D}$ (39).

\begin{tabular}{lllllll}
\hline & & $N=8$ & $N=16$ & $N=32$ & $N=64$ & $N=128$ \\
\hline \multirow{2}{*}{$\kappa=10^{-4}$} & $\left\|\boldsymbol{u}-\boldsymbol{u}_{h}\right\|_{A}$ & 0.0151 & 0.0072 & 0.0037 & 0.0019 & 0.0010 \\
& $\left\|p-p_{h}\right\|_{L^{2}}$ & 0.0322 & 0.0168 & 0.0104 & 0.0052 & 0.0020 \\
\hline \multirow{2}{*}{$\kappa=10^{-6}$} & $\left\|\boldsymbol{u}-\boldsymbol{u}_{h}\right\|_{A}$ & 0.0153 & 0.0073 & 0.0036 & 0.0018 & 0.0009 \\
& $\left\|p-p_{h}\right\|_{L^{2}}$ & 0.0349 & 0.0161 & 0.0074 & 0.0032 & 0.0018 \\
\hline \multirow{2}{*}{$\kappa=10^{-8}$} & $\left\|\boldsymbol{u}-\boldsymbol{u}_{h}\right\|_{A}$ & 0.0153 & 0.0073 & 0.0036 & 0.0035 & 0.00012 \\
\hline \multirow{2}{*}{$\kappa=10^{-10}$} & $\left\|p-p_{h}\right\|_{L^{2}}$ & 0.0349 & 0.0162 & 0.0074 & 0.0018 & 0.00035 \\
& $\left\|\boldsymbol{u}-\boldsymbol{u}_{h}\right\|_{A}$ & 0.0153 & 0.0073 & 0.0036 & 0.0075 & 0.0017 \\
\hline
\end{tabular}

Now, using estimates (65) and (66), we obtain the following a priori error estimate,

$$
\begin{aligned}
\left\|\boldsymbol{u}-\Pi_{h}^{\mathrm{BDM}} \boldsymbol{u}_{h}\right\| & \leq\left\|\boldsymbol{u}-\Pi_{h}^{\mathrm{BDM}} \boldsymbol{u}\right\|+\left\|\Pi_{h}^{\mathrm{BDM}}\left(\boldsymbol{u}-\boldsymbol{u}_{h}\right)\right\| \\
& \lesssim h|\boldsymbol{u}|_{1}+h\left|\boldsymbol{u}-\boldsymbol{u}_{h}\right|_{1}+\left\|\boldsymbol{u}-\boldsymbol{u}_{h}\right\| \\
& \lesssim h|\boldsymbol{u}|_{1}+\left|\boldsymbol{u}-\boldsymbol{u}_{h}\right|_{1} \lesssim h\|\boldsymbol{u}\|_{2} .
\end{aligned}
$$

Note that we have used Korn's inequality for $\left(\boldsymbol{u}-\boldsymbol{u}_{h}\right)$, which is a function vanishing on the Dirichlet part of the boundary. Thus, (64) and the a priori estimate above guarantee that the BDM interpolant of the numerical solution, $\Pi_{h}^{\mathrm{BDM}} \boldsymbol{u}_{\boldsymbol{h}}$, is a mass-conserving approximation to $\boldsymbol{u}$, which requires little extra cost to compute.

\section{Numerical results}

In this section we illustrate the theoretical convergence results obtained in previous sections. We present results for both the poroelastic problem and for Stokes' equations. All test problems were implemented in the HAZmath library [43], which contains routines for finite elements, multilevel solvers, and graph algorithms.

\subsection{Poroelastic problem}

First we consider the test included in Section 2.2, in order to show the corresponding results when the stabilized P1-RT0-P0 is considered. Table 6.1 displays the energy norm errors for displacement and $L^{2}$-norm errors for pressure obtained by applying the scheme after diagonalizing the block corresponding to the bubble functions, $\mathcal{A}^{D}$ (System (39)). For this test, different values of the parameter $\kappa$ and different mesh-sizes are considered to show that the errors are appropriately reduced independently of the physical parameters, in contrast to the original P1-RT0-P0 scheme (Table 2.1).

We also compare the obtained errors with those provided by the fully enriched element, $\mathcal{A}$ (System (23)), in order to see that the same error reduction is achieved. Fig. 6.1, displays a comparison of the displacement and pressure errors in the energy and $L^{2}$ norms, respectively, for different grid sizes. We choose $\kappa=10^{-8}$ here, though similar pictures are obtained for different values of $\kappa$. We observe that the slopes corresponding to both schemes are the same, although the scheme corresponding to the diagonal version provides slightly worse errors. However, this scheme, when the bubble block is eliminated, uses less degrees of freedom and is easily implemented from an already existing P1-RT0-P0 code.

To demonstrate the method on a realistic test problem, we also consider a three-dimensional footing problem (see [44]). The computational domain is a unit cube modeling a block of porous soil. A uniform load of intensity $3 \times 10^{4} \mathrm{~N} / \mathrm{m}^{2}$ is applied in a square of size $0.5 \times 0.5 \mathrm{~m}^{2}$ at the top of the domain. The base of the domain is assumed to be fixed while the rest of the domain is free to drain. For the material properties, the Lame coefficients are computed in terms of the Young modulus, $E$, and the Poisson ratio, $v: \lambda=\frac{E v}{(1-2 v)(1+v)}$ and $\mu=\frac{E}{1+2 v}$. We fix $E=3 \times 10^{4} \mathrm{~N} / \mathrm{m}^{2}$ and $v=0.45$ to simulate a somewhat stiff material, and consider $K=10^{-6} \boldsymbol{I}$.

Fig. 6.2 compares the solution of this footing problem using both the fully enriched element, $\mathcal{A}$ (System (23)), and the scheme after diagonalizing the block corresponding to the bubble functions, $\mathcal{A}^{D}$ (System (39)). For simplicity, 


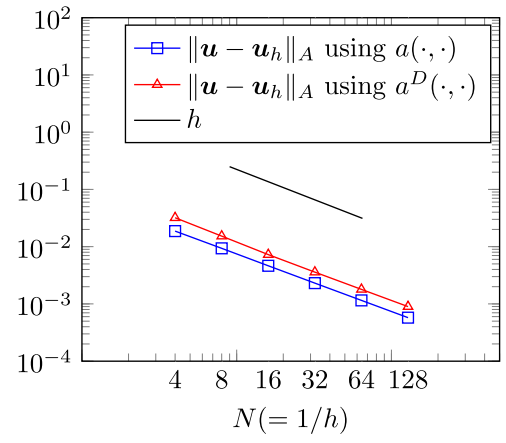

(a)

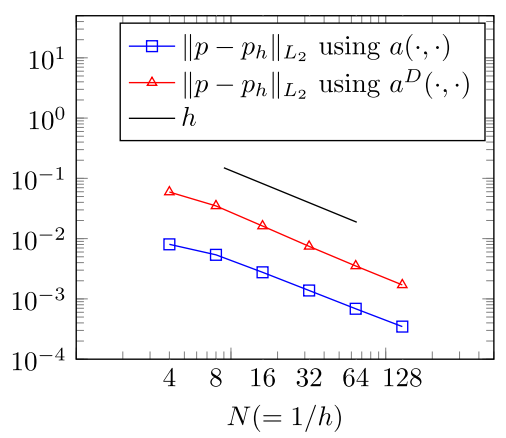

(b)

Fig. 6.1. Two-dimensional Biot's problem. Reduction of the (a) displacement and (b) pressure errors for different mesh-sizes, by using the enriched finite element scheme, $\mathcal{A}(23)$, as well as the scheme with diagonal block used for the bubble functions, $\mathcal{A}^{D}(39)$.

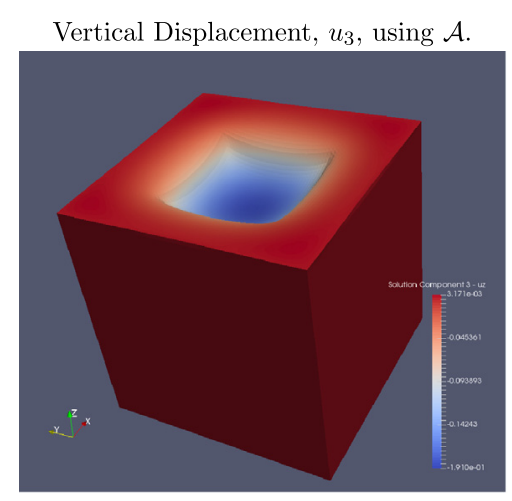

Pressure, $p$, using $\mathcal{A}$.

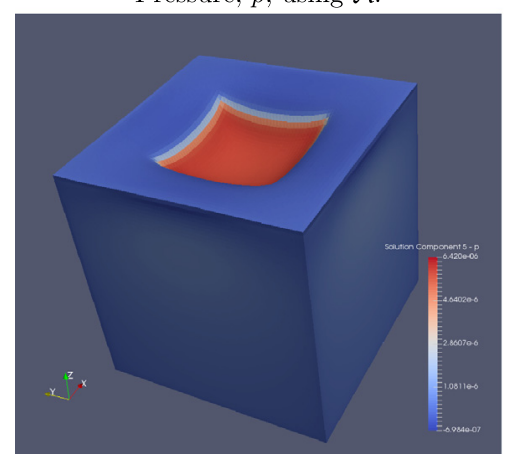

Vertical Displacement, $u_{3}$, using $\mathcal{A}^{D}$

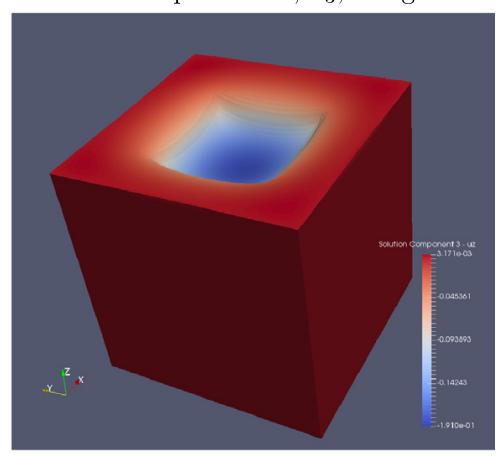

Pressure, $p$, using $\mathcal{A}^{D}$.

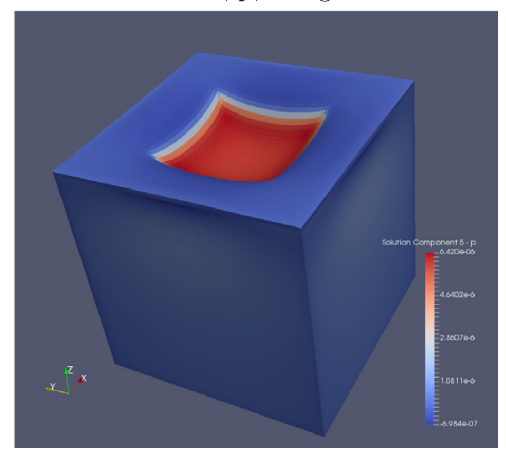

Fig. 6.2. Three-dimensional footing problem. Results are on a $64 \times 64 \times 64$ element grid.

we compare the vertical displacement and the pressure. We see little difference between the two sets of results. We confirm this by computing the difference in the two solutions using the energy norm for the displacements and the $L^{2}$ norm for pressure. Fig. 6.3 shows convergence of the two approaches with mesh size.

\subsection{Stokes' problem}

While it is well-known that the P1-P0 finite element pair is not stable for Stokes' equations, we show here that the new formulation, $\widehat{\mathcal{A}}_{S}^{D}(63)$, resulting from the elimination of the normal components of the bubbles, does provide a stable method. Consider (54)-(56) on a unit square $(0,1) \times(0,1)$, where the right-hand side $f$ is chosen such that the 


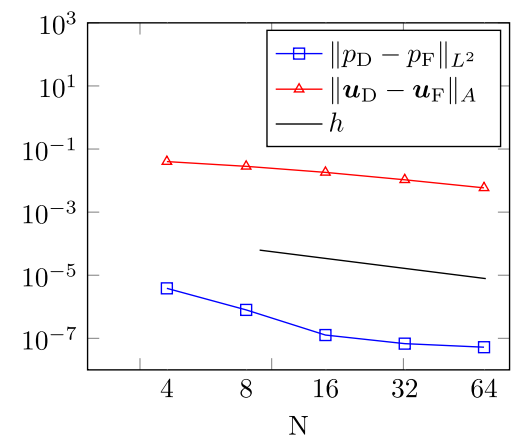

Fig. 6.3. Comparison of the displacement and pressure on the three-dimensional footing problem, using the fully-enriched formulation, $\mathcal{A}$ (23) (subscript $F$ ) and the scheme with diagonal block used for the bubble functions, $\mathcal{A}^{D}$ (39) (subscript $D$ ).

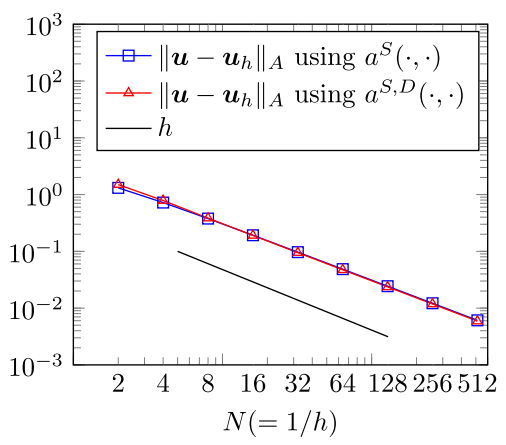

(a)

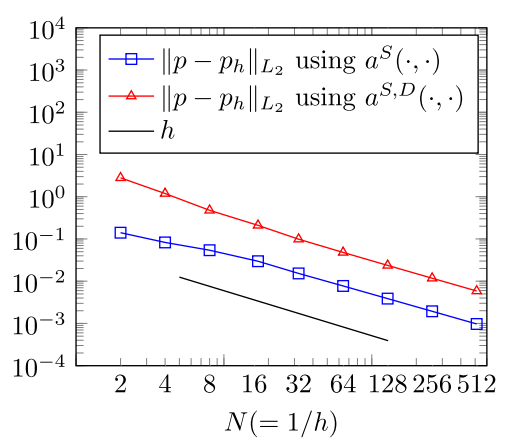

(b)

Fig. 6.4. Two-dimensional Stokes' problem. Reduction of the (a) velocity and (b) pressure errors for different mesh-sizes, by using the enriched finite element scheme, $\mathcal{A}_{S}(61)$, as well as the scheme resulting from using a diagonal block for the bubble functions, $\mathcal{A}_{S}^{D}$ (62).

analytical solution is given by

$$
\boldsymbol{u}(x, y)=(\sin (\pi x) \cos (\pi y),-\cos (\pi x) \sin (\pi y)), \quad p(x, y)=0.5-x .
$$

Fig. 6.4 compares the error reduction for both the velocity and pressure using the bubble function enhanced schemes described by $\mathcal{A}_{S}(61)$ and $\mathcal{A}_{S}^{D}(62)$.

We perform a similar three-dimensional test, solving (54)-(56) on a unit cube, where the right-hand side $f$ is chosen such that the analytical solution is given by

$$
\boldsymbol{u}(x, y)=(-\sin (\pi x) \sin (\pi(y-z)), \sin (\pi y) \sin (\pi(x-z)),-\sin (\pi z) \sin (\pi(x-y))), \quad p(x, y)=0.5-x .
$$

The results are shown in Fig. 6.5.

For all test problems, the energy norm for the velocity is defined as $\|\boldsymbol{v}\|_{A}^{2}:=a^{S}(\boldsymbol{v}, \boldsymbol{v})=2 \mu(\boldsymbol{\varepsilon}(\boldsymbol{v}), \boldsymbol{\varepsilon}(\boldsymbol{v}))$ for $\boldsymbol{v} \in \boldsymbol{V}$. Both methods give the same, optimal, order of convergence, demonstrating that the inclusion of the bubble functions guarantee the stability of the method. Moreover, though the errors are slightly higher, the elimination of the bubble functions would provide a stable convergent method, but reduces the problem to one that contains the same number of degrees of freedom as the $\mathrm{P} 1-\mathrm{P} 0$ discretization. Thus, we get a stable scheme with no increase in cost.

\section{Conclusions}

In this paper, we have shown how to stabilize the popular P1-RT0-P0 finite-element discretization for a three-field formulation of the poroelasticity problem. By adding the normal components of the bubble basis functions associated with the faces of the triangulation to the P1 element for displacements, we have demonstrated that an inf-sup condition is satisfied independently of the physical and discretization parameters of the problem. Moreover, the degrees of 


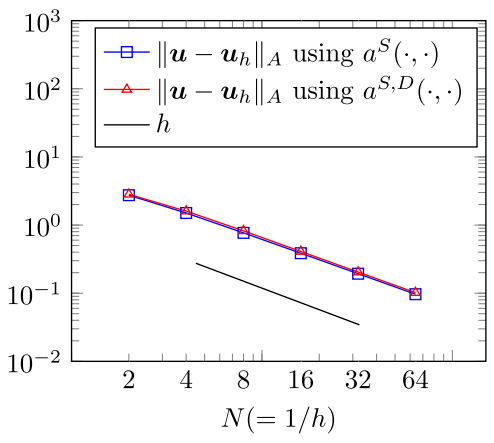

(a)

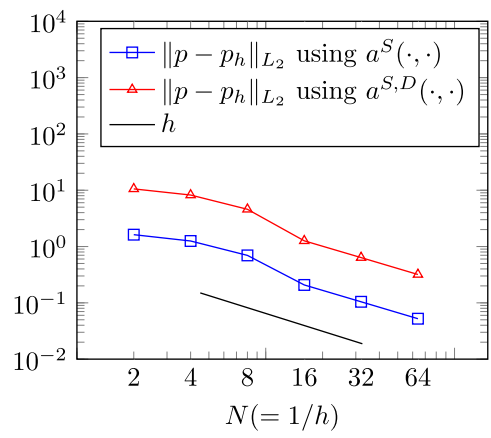

(b)

Fig. 6.5. Three-dimensional Stokes' problem. Reduction of the (a) velocity and (b) pressure errors for different mesh-sizes, by using the enriched finite element scheme, $\mathcal{A}_{S}$ (61), as well as the scheme resulting from using a diagonal block for the bubble functions, $\mathcal{A}_{S}^{D}$ (62).

freedom added to the faces can be eliminated resulting in a stable scheme with the same number of unknowns as in the initial P1-RT0-P0 discretization. Furthermore, this idea has been extended to the Stokes' equations, yielding a stable finite-element formulation with the lowest possible number of degrees of freedom, equivalent to a P1-PO discretization. Future work includes investigating such formulations and their performance for various applications in poroelasticity, and extending the discretization to other PDE systems which have similar properties to the Stokes' equations.

\section{Acknowledgments}

The work of F.J. Gaspar was supported by the European Union's Horizon 2020 research and innovation programme under the Marie Sklodowska-Curie grant agreement No. 705402, POROSOS. The research of C. Rodrigo was supported in part by the Spanish project FEDER/MCYT MTM2016-75139-R and the DGA (Grupo de referencia APEDIF, ref. E24-17R). The work of L.T. Zikatanov was partially supported by NSF grants DMS-1522615 and DMS-1720114. The work of J.H. Adler, X. Hu and P. Ohm was partially supported by NSF grant DMS-1620063.

\section{References}

[1] K. Terzaghi, Theoretical Soil Mechanics, Wiley: New York, 1943.

[2] M.A. Biot, General Theory of three-dimensional consolidation, J. Appl. Phys. 12 (2) (1941) 155-164.

[3] M.A. Biot, Theory of elasticity and consolidation for a porous anisotropic solid, J. Appl. Phys. 26 (2) (1955) $182-185$.

[4] R. Showalter, Diffusion in poro-elastic media, J. Math. Anal. Appl. 251 (1) (2000) 310-340.

[5] A. Ženíšek, The existence and uniqueness theorem in Biot's consolidation theory, Apl. Mat. 29 (3) (1984) $194-211$.

[6] F.J. Gaspar, F.J. Lisbona, P.N. Vabishchevich, A finite difference analysis of Biot's consolidation model, Appl. Numer. Math. 44 (4) (2003) 487-506. http://dx.doi.org/10.1016/S0168-9274(02)00190-3.

[7] F.J. Gaspar, F.J. Lisbona, P.N. Vabishchevich, Staggered grid discretizations for the quasi-static Biot's consolidation problem, Appl. Numer. Math. 56 (6) (2006) 888-898. http://dx.doi.org/10.1016/j.apnum.2005.07.002.

[8] J.M. Nordbotten, Cell-centered finite volume discretizations for deformable porous media, Internat. J. Numer. Methods Engrg. 100 (6) (2014) $399-418$.

[9] J.M. Nordbotten, Stable cell-centered finite volume discretization for biot equations, SIAM J. Numer. Anal. 54 (2) (2016) $942-968$.

[10] R. Lewis, B. Schrefler, The Finite Element Method in the Static and Dynamic Deformation and Consolidation of Porous Media, Wiley: New York, 1998.

[11] M.A. Murad, A.F.D. Loula, Improved accuracy in finite element analysis of Biot's consolidation problem, Comput. Methods Appl. Mech. Engrg. 95 (3) (1992) 359-382. http://dx.doi.org/10.1016/0045-7825(92)90193-N.

[12] M.A. Murad, A.F.D. Loula, On stability and convergence of finite element approximations of Biot's consolidation problem, Internat. J. Numer. Methods Engrg. 37 (4) (1994) 645-667. http://dx.doi.org/10.1002/nme.1620370407.

[13] M.A. Murad, V. Thomée, A.F.D. Loula, Asymptotic behavior of semidiscrete finite-element approximations of Biot's consolidation problem, SIAM J. Numer. Anal. 33 (3) (1996) 1065-1083. http://dx.doi.org/10.1137/0733052.

[14] C. Rodrigo, F. Gaspar, X. Hu, L. Zikatanov, Stability and monotonicity for some discretizations of the Biot's consolidation model, Comput. Methods Appl. Mech. Engrg. 298 (2016) 183-204.

[15] X. Hu, C. Rodrigo, F.J. Gaspar, L.T. Zikatanov, A nonconforming finite element method for the Biot's consolidation model in poroelasticity, J. Comput. Appl. Math. 310 (2017) 143-154. http://dx.doi.org/10.1016/j.cam.2016.06.003. 
[16] Q. Hong, J. Kraus, Parameter-robust stability of classical three-field formulation of Biot's consolidation model, Electron. Trans. Numer. Anal. 48 (2018) 202-226.

[17] R. Oyarzúa, R. Ruiz-Baier, Locking-free finite element methods for poroelasticity, SIAM J. Numer. Anal. 54 (5) (2016) $2951-2973$.

[18] J.J. Lee, K.-A. Mardal, R. Winther, Parameter-robust discretization and preconditioning of Biot's consolidation model, SIAM J. Sci. Comput. 39 (1) (2017) A1-A24.

[19] J.J. Lee, Robust error analysis of coupled mixed methods for biot's consolidation model, J. Sci. Comput. 69 (2) (2016) 610-632.

[20] A. Mikelić, M.F. Wheeler, Convergence of iterative coupling for coupled flow and geomechanics, Comput. Geosci. 17 (3) (2013) 455-461.

[21] J.W. Both, M. Borregales, J.M. Nordbotten, K. Kumar, F.A. Radu, Robust fixed stress splitting for Biot's equations in heterogeneous media, Appl. Math. Lett. 68 (2017) 101-108.

[22] P. Phillips, M. Wheeler, A coupling of mixed and continuous Galerkin finite element methods for poroelasticity I: the continuous in time case, Comput. Geosci. 11 (2) (2007) 131-144. http://dx.doi.org/10.1007/s10596-007-9045-y.

[23] P. Phillips, M. Wheeler, A coupling of mixed and continuous Galerkin finite element methods for poroelasticity II: the discrete-in-time case, Comput. Geosci. 11 (2) (2007) 145-158. http://dx.doi.org/10.1007/s10596-007-9044-z.

[24] P. Phillips, M. Wheeler, A coupling of mixed and discontinuous Galerkin finite-element methods for poroelasticity, Comput. Geosci. 12 (4) (2008) 417-435. http://dx.doi.org/10.1007/s10596-008-9082-1.

[25] N. Castelleto, J.A. White, M. Ferronato, Scalable algorithms for three-field mixed finite element coupled poromechanics, J. Comput. Phys. 327 (2016) 894-918.

[26] M. Bause, F. Radu, U. Kocher, Space-time finite element approximation of the Biot poroelasticity system with iterative coupling, Comput. Methods Appl. Mech. Engrg. 320 (2017) 745-768. http://dx.doi.org/10.1016/j.cma.2017.03.017.

[27] T. Almani, K. Kumar, A. Dogru, G. Singh, M. Wheeler, Convergence analysis of multirate fixed-stress split iterative schemes for coupling flow with geomechanics, Comput. Methods Appl. Mech. Engrg. 311 (1) (2016) 180-207.

[28] K. Lipnikov, Numerical methods for the Biot model in poroelasticity, Ph.D. thesis, University of Houston, 2002.

[29] F. Brezzi, M. Fortin, Mixed and hybrid finite element methods, in: Springer Series in Computational Mathematics, vol.15, Springer-Verlag, New York, 1991, p. x+350. http://dx.doi.org/10.1007/978-1-4612-3172-1.

[30] D. Boffi, F. Brezzi, L.F. Demkowicz, R.G. Durán, R.S. Falk, M. Fortin, Mixed finite elements, compatibility conditions, and applications, in: Lecture Notes in Mathematics, vol.1939, Springer-Verlag, Berlin; Fondazione C.I.M.E., Florence, 2008, p. x+235. http://dx.doi.org/10. 1007/978-3-540-78319-0. Lectures given at the C.I.M.E. Summer School held in Cetraro, June 26-July 1, 2006, Edited by Boffi and Lucia Gastaldi.

[31] D. Boffi, F. Brezzi, M. Fortin, Mixed finite element methods and applications, in: Springer Series in Computational Mathematics, vol. 44, Springer, Heidelberg, 2013, p. xiv+685. http://dx.doi.org/10.1007/978-3-642-36519-5.

[32] V. Girault, P.-A. Raviart, Finite element methods for Navier-Stokes equations, in: Springer Series in Computational Mathematics, vol. 5, Springer-Verlag, Berlin, 1986, p. x+374. http://dx.doi.org/10.1007/978-3-642-61623-5. Theory and algorithms.

[33] X. Hu, C. Rodrigo, F.J. Gaspar, L.T. Zikatanov, A nonconforming finite element method for the Biot's consolidation model in poroelasticity, J. Comput. Appl. Math. 310 (2017) 143-154. http://dx.doi.org/10.1016/j.cam.2016.06.003.

[34] A. Korn, Solution general du probleme d'equilibre dans la theorie de l'elasticite, Ann. Fac. Sci. Toulouse 10 (1908) $705-724$.

[35] A. Korn, Ueber einige Ungleichungen, welche in der Theorie der elastischen und elektrischen Schwingungen eine Rolle spielen, Bull. Int. Acad. Sci. Crac. 9 (1909) 705-724.

[36] V.A. Kondratiev, O.A. Oleinik, On Korn's inequalities, C. R. Acad. Sci. Paris Sé. I Math. 308 (16) (1989) 483-487. http://dx.doi.org/10.1070/ RM1989v044n06ABEH002297.

[37] V.A. Kondratiev, O.A. Oleinik, Dependence of the constants in the Korn inequality on parameters that characterize the geometry of the domain, Uspekhi Mat. Nauk 44 (6(270)) (1989) 157-158. http://dx.doi.org/10.1070/RM1989v044n06ABEH002297.

[38] P.G. Ciarlet, The finite element method for elliptic problems, North-Holland Publishing Co., Amsterdam-New York-Oxford, 1978, p. xix+530 Studies in Mathematics and its Applications, Vol. 4.

[39] V. Thomée, Galerkin finite element methods for parabolic problems, second ed., in: Springer Series in Computational Mathematics, vol. 25, Springer-Verlag, Berlin, 2006, p. xii+370.

[40] F. Brezzi, Jr., J. Douglas, L.D. Marini, Recent results on mixed finite element methods for second order elliptic problems, in: Vistas in Applied Mathematics, in: Transl. Ser. Math. Engrg., Optimization Software, New York, 1986, pp. 25-43.

[41] F. Brezzi, Jr., J. Douglas, R. Durán, M. Fortin, Mixed finite elements for second order elliptic problems in three variables, Numer. Math. 51 (2) (1987) 237-250. http://dx.doi.org/10.1007/BF01396752.

[42] P. Monk, Finite element methods for Maxwell's equations, Numerical Mathematics and Scientific Computation, Oxford University Press, New York, 2003, p. xiv+450. http://dx.doi.org/10.1093/acprof:oso/9780198508885.001.0001.

[43] X. Hu, J.H. Adler, L.T. Zikatanov, HAZmath: A Simple Finite Element, Graph, and Solver Library, 2014-2017 https://bitbucket.org/Xiaozhe Hu/hazmath/wiki/Home.

[44] F.J. Gaspar, J.L. Gracia, F.J. Lisbona, C.W. Oosterlee, Distributive smoothers in multigrid for problems with dominating grad-div operators, Numer. Linear Algebra Appl. 15 (8) (2008) 661-683. http://dx.doi.org/10.1002/nla.587. 